\title{
Limit cycles of discontinuous piecewise linear differential systems formed by centers or Hamiltonian without equilibria separated by irreducible cubics
}

\section{Loubna DAMEnE ${ }^{1}$ AND Rebiha Benterki ${ }^{2}$}

Aвstract. The main goal of this paper is to provide the maximum number of crossing limit cycles of two different families of discontinuous piecewise linear differential systems. More precisely we prove that the systems formed by two regions, where, in one region we define a linear center and in the second region we define a Hamiltonian system without equilibria can exhibit three crossing limit cycles having two or four intersection points with the cubic of separation. After we prove that the systems formed by three regions, where, in two noadjacent regions we define a Hamiltonian system without equilibria, and in the third region we define a center, can exhibit six crossing limit cycles having four and two simultaneously intersection points with the cubic of separation.

Mathematics Subject Classification (2020). 34C29, 34C25, 47H11.

Key words and phrases. Limit cycles, Discontinuous piecewise linear differential systems, Linear centers, Linear Hamiltonian systems, Irreducible cubic curves.

\section{Introduction}

1.1. Classification of the irreducible cubics. An algebraic cubic curve or simple a cubic is the set of points $(x, y) \in \mathbb{R}^{2}$ satisfying $P(x, y)=0$ for some polynomial $P(x, y)$ of degree three.

Received : July 07, 2020 - Accepted: December 23, 2020.

(C)The Author(s) 2020. This article is published with open access by Sidi Mohamed Ben Abdallah University.

1,2 Département de Mathématiques, Université Mohamed El Bachir El Ibrahimi, Bordj Bou Arréridj 34000, El Anasser, Algeria.

e-mail : loubna.damene@univ-bba.dz

e-mail²: r.benterki@univ-bba.dz (Corresponding Author). 
This real cubic is irreducible (respectively reducible) if the polynomial $P(x, y)$ is irreducible (respectively reducible) in the ring of all real polynomials in the variables $x$ and $y$.

A point $\left(x_{0}, y_{0}\right)$ of a cubic $P(x, y)=0$ is singular if $P_{x}\left(x_{0}, y_{0}\right)=P_{y}\left(x_{0}, y_{0}\right)=0$. A cubic curve is singular if it has some singular point.

A flex of an algebraic curve $C$ is a point $p$ of $C$ such that $C$ is nonsingular at $p$ and the tangent at $p$ of the curve $C$ intersects $C$ at least three times.

Theorem 1.1. The following statements classify all the irreducible cubic algebraic curves.

(a) A cubic is nonsingular and irreducible if and only if it can be transformed with an affine transformation into one of the following two curves

$$
\begin{array}{ll}
c_{1}=c_{1}(x, y)=y^{2}-x\left(x^{2}+b x+1\right)=0 & \text { with } b \in(-2,2), \text { or } \\
c_{2}=c_{2}(x, y)=y^{2}-x(x-1)(x-r)=0 & \text { with } r>1 .
\end{array}
$$

(b) A cubic is singular and irreducible if and only if it can be transformed with an affine transformation into one of the following three curves:

$$
\begin{array}{ll}
c_{3}=c_{3}(x, y)=y^{2}-x^{3}=0, & \text { or } \\
c_{4}=c_{4}(x, y)=y^{2}-x^{2}(x-1)=0, & \text { or } \\
c_{5}=c_{5}(x, y)=y^{2}-x^{2}(x+1)=0 . &
\end{array}
$$

Statement (a) of Theorem 1.1 is proved in Theorem 8.3 of the book [7] under the additional assumption that the cubic has a flex, but in section 12 of that book it is shown that every nonsingular irreducible cubic curve has a flex. While statement (b) of Theorem 1.1 follows directly from Theorem 8.4 of [7].

Figures 1 and 2 show the different regions separated by the cubic curves $c_{i}=0$, with $i=$ $1, \ldots 5$.
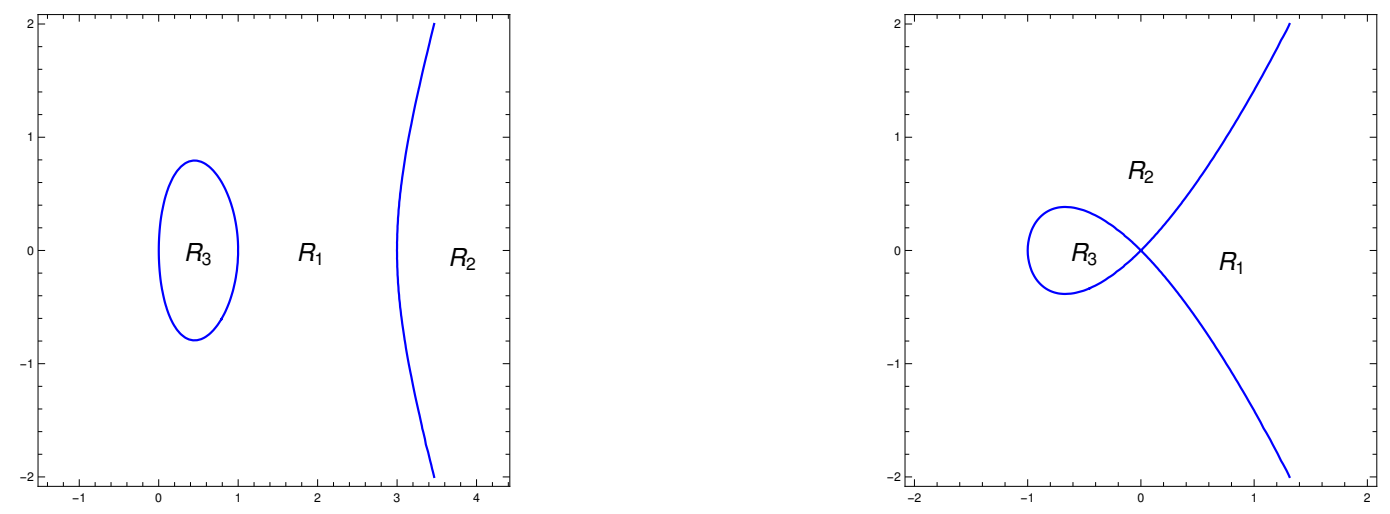

FIGURE 1. The three regions $R_{1}, R_{2}$ and $R_{3}$ of the plane separated by the curves $c_{2}=0$ on the left and $c_{5}=0$ on the right. 

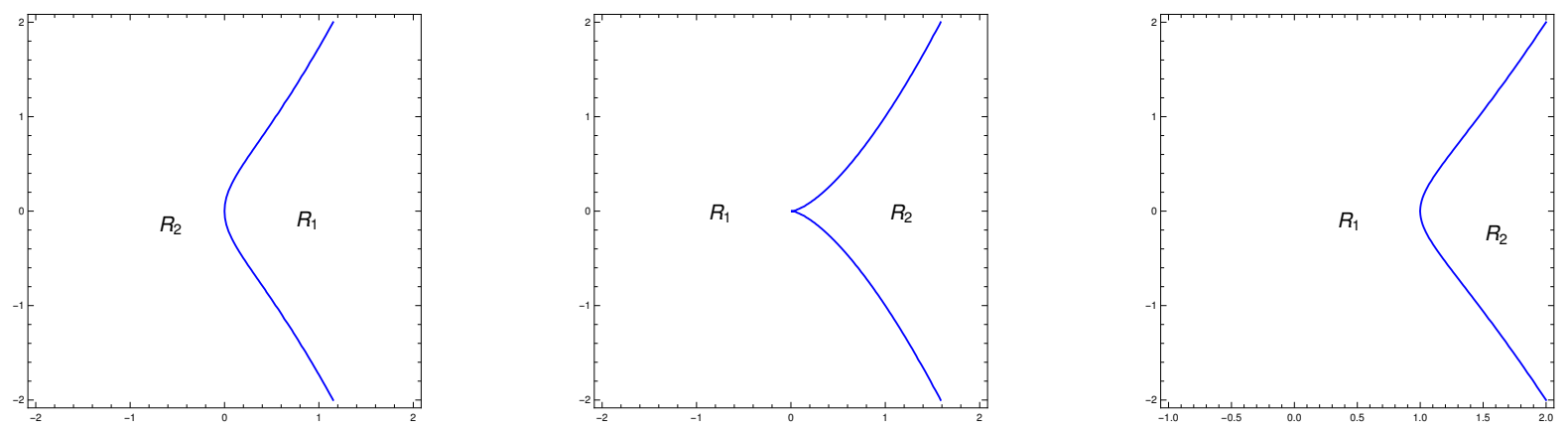

Figure 2. The two regions $R_{1}$ and $R_{2}$ of the plane separated by the curves $c_{1}=0$ on the left, $c_{3}=0$ on the middle and $c_{4}=0$ on the right.

1.2. Crossing limit cycles. The second part of the 16-th Hilber problem asks for the maximum number of limit cycles and their possible configuration of planar polynomial vector field of degree $n$. We know that, planar polynomial differential systems have no limit cycles, while it is not the case for piecewise linear differential systems. These systems revent to Andronov, Vitt and Khaikin [1]. Owing to the simplicity of this kind of differential systems, researchers had given a big interest on studying them and they have extensively a large relevance in the domain of engineering sciences, for example, we can model key of component in even simple electronic circuit, also the Diodes and transistors as a piecewise linear differential systems, etc., see for instance the survey of Makarenkov and Lamb [20], and the books of Bernardo and Simpson [21]. Even now, the 16-th Hilbert problem still open for this type of systems. We recall that a limit cycle of a differential system is an isolated periodic orbit in the set of all periodic orbit of this system.

Recently, many authors have been interesting to solve the second part of Hilbert 16-th problem for these systems in $\mathbb{R}^{2}$ when we separate them by a curve $\Sigma$. In [14] authors proved that if $\Sigma$ is a straight line then discontinuous piecewise linear differential centers have no crossing limit cycle. There are many other papers devoted to study the existence and the number of limit cycles of these systems when the curve of separation is a straight line, see for instance $[2,8,9,10,11,12,15,18]$.

In $[3,4,13]$ authors studied the number of crossing limit cycles of discontinuous piecewise linear differential centers separated by an algebraic curve, such that a conic, reducible or irreducible cubic curve.

For another type of planar piecewise linear differential systems, especially Hamiltonian systems without equilibrium points, Benterki and Llibre in [5] proved that if these systems separated by a parabola, a hyperbola or an ellipse; they can have at most 2, 3 or 3 crossing limit cycles, respectively.

In our paper we are going to consider two families of planar linear differential systems, the first one is Hamiltonians without equilibrium points and the second one is a family of centers, such that 
I. Any linear, differential, vector fields of Hamiltonian systems without equilibrium points takes the following form

$$
X(x, y)=\left(-\lambda b_{1} x+b_{1} y+\mu,-\lambda^{2} b_{1} x+\lambda b_{1} y+\sigma\right),
$$

$\sigma \neq \lambda \mu$ and $b_{1} \neq 0$. Its corresponding Hamiltonian function is

$$
H(x, y)=\left(-\lambda^{2} b_{1} / 2\right) x^{2}+\lambda b_{1} x y-\left(b_{1} / 2\right) y^{2}+\sigma x-\mu y .
$$

For more details see [10].

II. For the second family we will use the following lemma which provides a normal form for an arbitrary linear differential center. See a proof in [18].

Lemma 1.1. Through a linear change of variables and a rescaling of the independent variable every center in $\mathbb{R}^{2}$ can be written

$$
\dot{x}=-b_{2} x-\frac{4 b_{2}^{2}+\omega^{2}}{4} y+d, \quad \dot{y}=x+b_{2} y+c, \text { with } \omega>0 .
$$

The first integral of this linear differential system is

$$
H(x, y)=4\left(x+b_{2} y\right)^{2}+8(c x-d y)+y^{2} \omega^{2} .
$$

1.3. Statement of the main results. We denote by $\mathcal{F}_{1}$ the family of discontinuous piecewise differential systems separated by a cubic curve $c_{k}=0$ for $k=1, \ldots, 5$ contained in two regions. We note that such piecewise systems formed by two pieces, in one piece we define a linear differential center, and in the other piece we define a linear Hamiltonian system without equilibrium point.

Now we denote by $\mathcal{F}_{2}$ the family of discontinuous piecewise differential systems separated by a cubic curve $c_{2}=0$ or $c_{5}=0$ contained in three regions. When the cubic of separation is $c_{2}=0$, we define in regions $R_{2}$ and $R_{3}$ a Hamiltonian system without equilibrium point, and we define in the region $R_{1}$ a linear center. When the cubic of separation is $c_{5}=0$, we define in regions $R_{1}$ and $R_{3}$ a Hamiltonian system without equilibrium points, and in the region $R_{2}$ we define a linear center.

We denote by $C_{k}$ the class of planar discontinuous piecewise differential systems of family $\mathcal{F}_{1}$ or $\mathcal{F}_{2}$ separated by irreducible cubic curve $c_{k}=0$ for $k=1, \ldots, 5$.

Our first objective in this work is to provide the maximum number of crossing limit cycles of the family $\mathcal{F}_{1}$ which intersect the irreducible cubic curves $c_{i}=0$, with $i=1 \ldots 5$ in two points.

The notation $\left(C_{k}^{i}\right)$ indicate the configuration number $i$ of crossing limit cycle for the class $C_{k}$, where $i \in\{1,2,3,4,5,6,7\}$ and $k=2, \ldots, 5$.

Then our first main result is the following.

Theorem 1.2. The maximum number of crossing limit cycles of discontinuous piecewise differential systems of the family $\mathcal{F}_{1}$ which intersect the cubic curve $c_{k}=0$, for $k=1, \ldots, 5$ in two points is three. This maximum is reached in all cases.

(i) For classes $C_{1}, C_{3}$ and $C_{4}$, see Examples 1, 2 and 3, respectively.

(ii) For the class $C_{2}$, see Examples 4.1, 4.2, 4.3, 4.4, 4.5, 4.6 and 4.7.

(iii) For the class $C_{5}$, see Examples 5.1, 5.2, 5.3, 5.4, 5.5, 5.6 and 5.7. 
Theorem 1.2 is proved in section 2 .

The second objective of this work is to provide the maximum number of crossing limit cycles of the family $\mathcal{F}_{2}$ which intersect the irreducible cubic curve $c_{2}=0$ or $c_{5}=0$ in four points or in four and two points simultaneously. Then we have the following result.

Theorem 1.3. The following statements hold.

(a) The maximum number of crossing limit cycles of systems of the family $\mathcal{F}_{2}$ which intersect the cubic curve $c_{2}=0$ or $c_{5}=0$ in four points is three. See Example 6 for the class $C_{2}$ and Example 7 for the class $C_{5}$.

(b) The maximum number of crossing limit cycles of discontinuous piecewise differential systems of the family $\mathcal{F}_{2}$ intersecting simultaneously in four points and two points the cubic $c_{2}=0$ or $c_{5}=0$ is six.

This maximum is reached, see Example 8 for the class $C_{2}$ and Example 9 for the class $C_{5}$, where there are systems exhibiting three limit cycle with four intersection points and three limit cycles with two intersection points with the cubic.

Theorem 1.3 is proved in section 3.

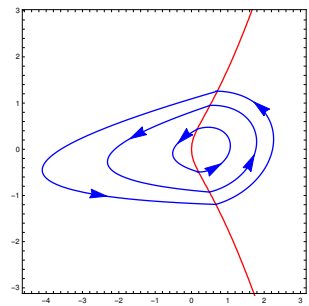

FiguRE 3. The three limit cycles of the discontinuous piecewise differential system (2.5)-(2.6).

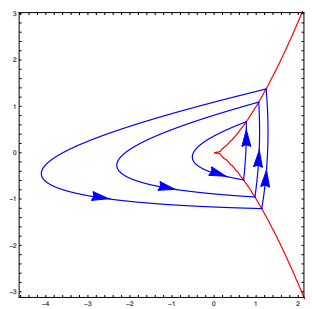

Figure 4 . The three limit cycles of the discontinuous piecewise differential system (2.7)-(2.8).

\section{Proof of Theorem 1.2}

In this section we have to prove that the maximum number of limit cycles of systems of family $\mathcal{F}_{1}$ intersecting the cubic curve $c_{3}=0$, in two points is three. By a similar way we prove the statement for the other four cubic curves. 


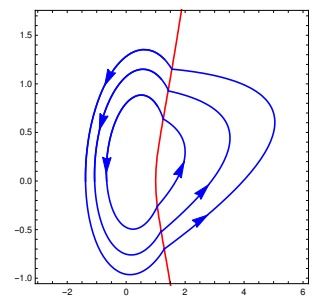

Figure 5. The three limit cycles of the discontinuous piecewise differential system (2.9)-(2.10).

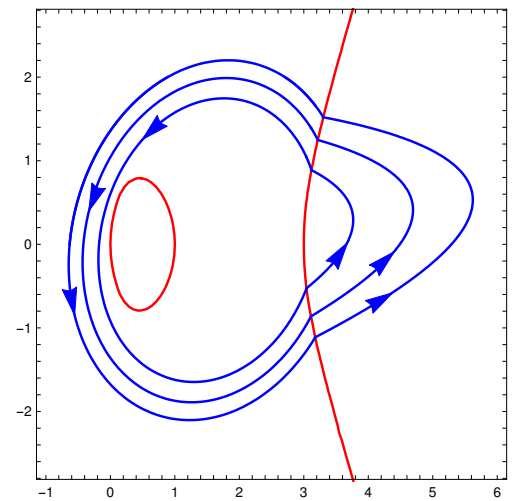

$\left(C_{2}^{1}\right)$

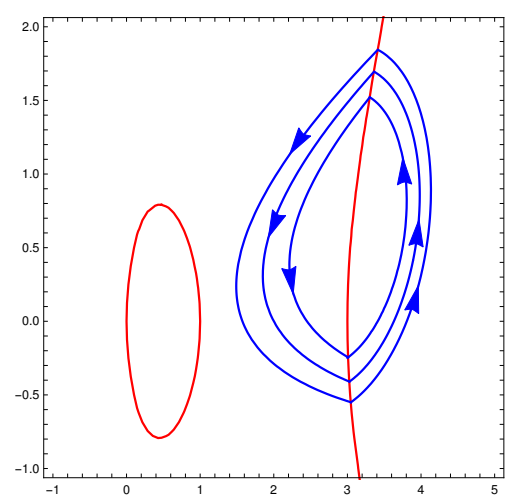

$\left(C_{2}^{2}\right)$

Figure 6. The three limit cycles of the discontinuous piecewise differential system $\left(C_{2}^{1}\right)$ for (2.12)-(2.13), and $\left(C_{2}^{2}\right)$ for (2.14)-(2.15)

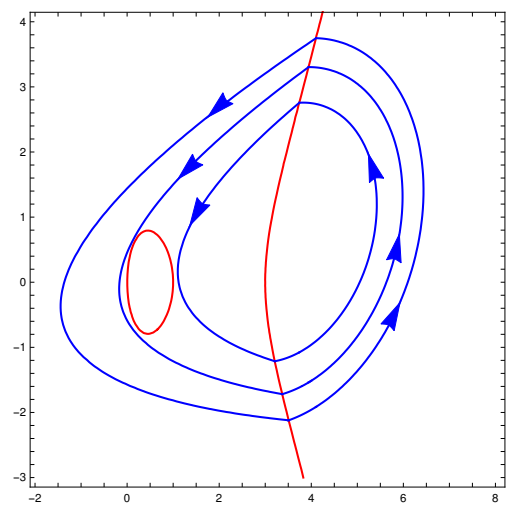

$\left(C_{2}^{3}\right)$

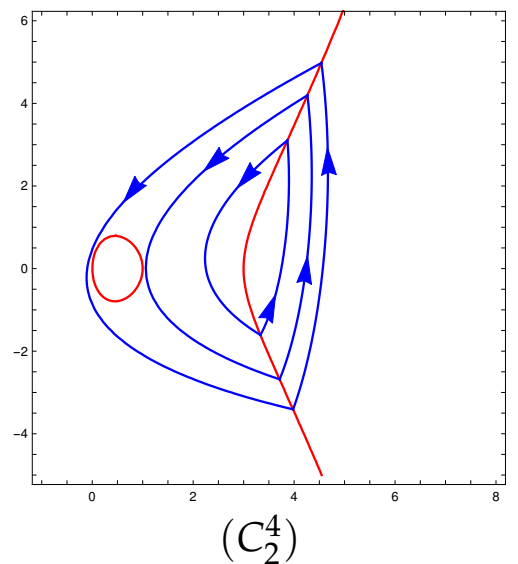

$\left(C_{2}^{4}\right)$

Figure 7. The three limit cycles of the discontinuous piecewise differential system $\left(C_{2}^{3}\right)$ for (2.16)-(2.17), and $\left(C_{2}^{4}\right)$ for (2.18)-(2.19)

We consider the discontinuous piecewise linear Hamiltonian system such that in the region $R_{1}=\left\{(x, y): y^{2}-x^{3} \geq 0\right\}$ is defined as

$$
\dot{x}=-\lambda b_{1} x+b_{1} y+\mu, \quad \dot{y}=-\lambda^{2} b_{1} x+\lambda b_{1} y+\sigma,
$$



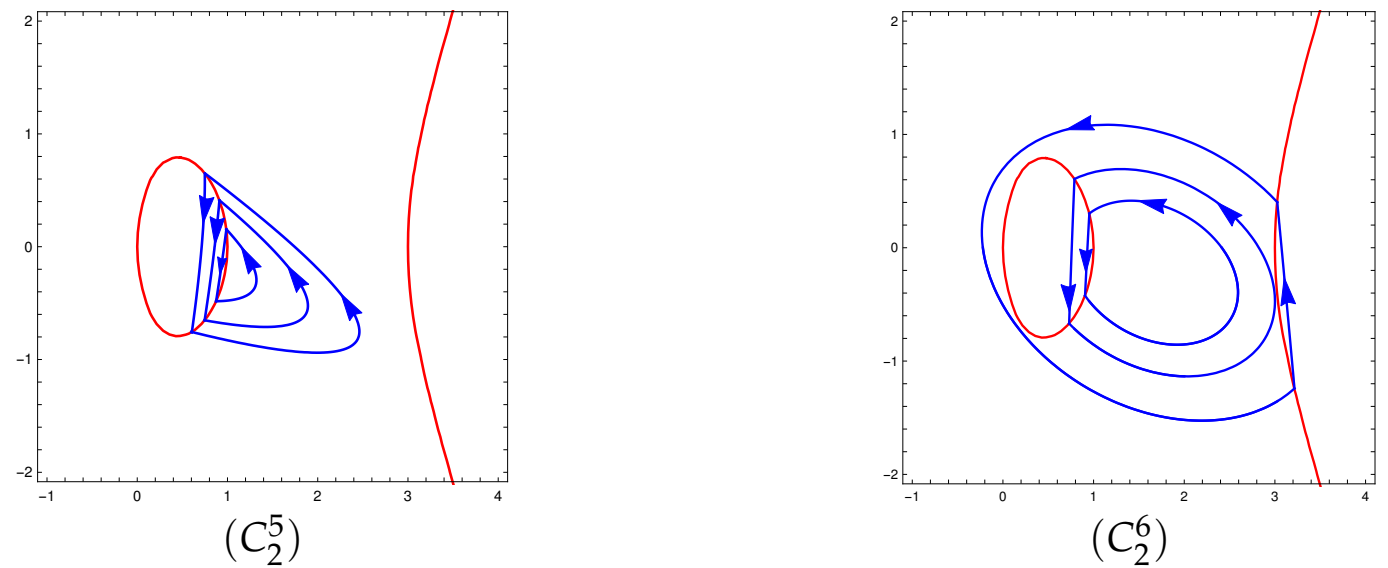

FIGURE 8. The three limit cycles of the discontinuous piecewise differential system $\left(C_{2}^{5}\right)$ for $(2.20)-(2.21)$, and $\left(C_{2}^{6}\right)$ for $(2.22)-(2.23)$

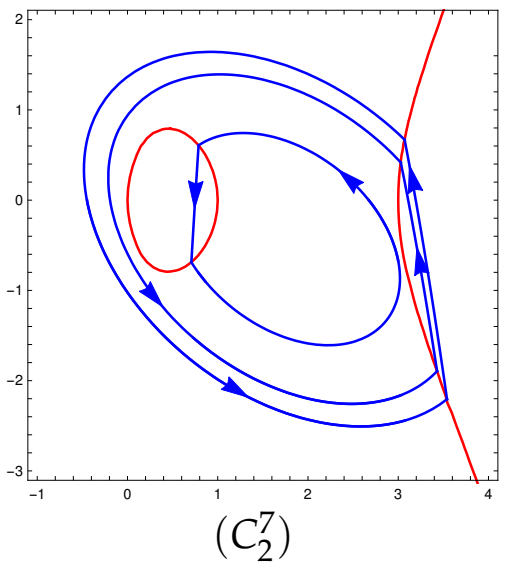

FigurE 9. The three limit cycles of the discontinuous piecewise differential system (2.24)-(2.25)

with $b_{1} \neq 0$ and $\sigma \neq \lambda \mu$. This system has the first integral

$$
H_{1}(x, y)=-\left(\lambda^{2} b_{1} / 2\right) x^{2}+\lambda b_{1} x y-\left(b_{1} / 2\right) y^{2}+\sigma x-\mu y .
$$

In the region $R_{2}=\left\{(x, y): y^{2}-x^{3} \leq 0\right\}$ we consider the linear center

$$
\dot{x}=-b_{2} x-\frac{4 b_{2}^{2}+\omega^{2}}{4} y+d, \quad \dot{y}=x+b_{2} y+c, \text { with } \omega>0 .
$$

Its first integral is

$$
H_{2}(x, y)=4\left(x+b_{2} y\right)^{2}+8(c x-d y)+y^{2} \omega^{2} .
$$

We have to prove that the piecewise linear differential systems composed by system (2.1) and system (2.2) have at most three crossing limit cycles intersecting the cubic curve $y^{2}-x^{3}=0$ in two points. 

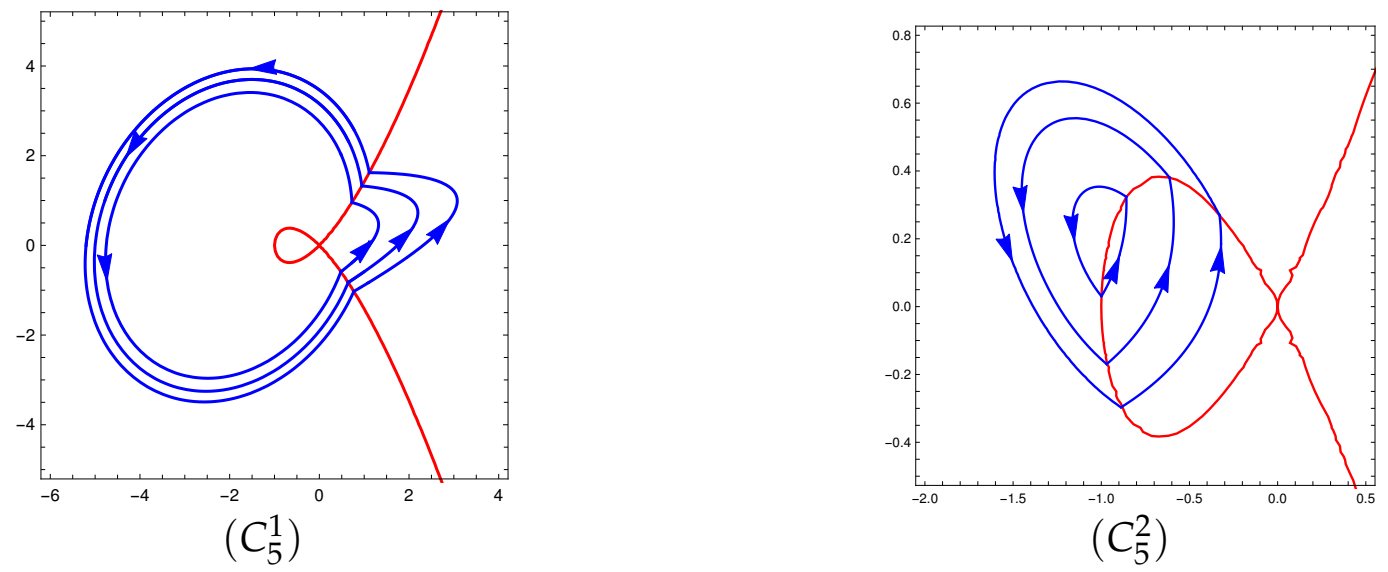

FIGURE 10. The three limit cycles of the discontinuous piecewise differential system $\left(C_{5}^{1}\right)$ for $(2.27)-(2.28)$, and $\left(C_{5}^{2}\right)$ for $(2.29)-(2.30)$
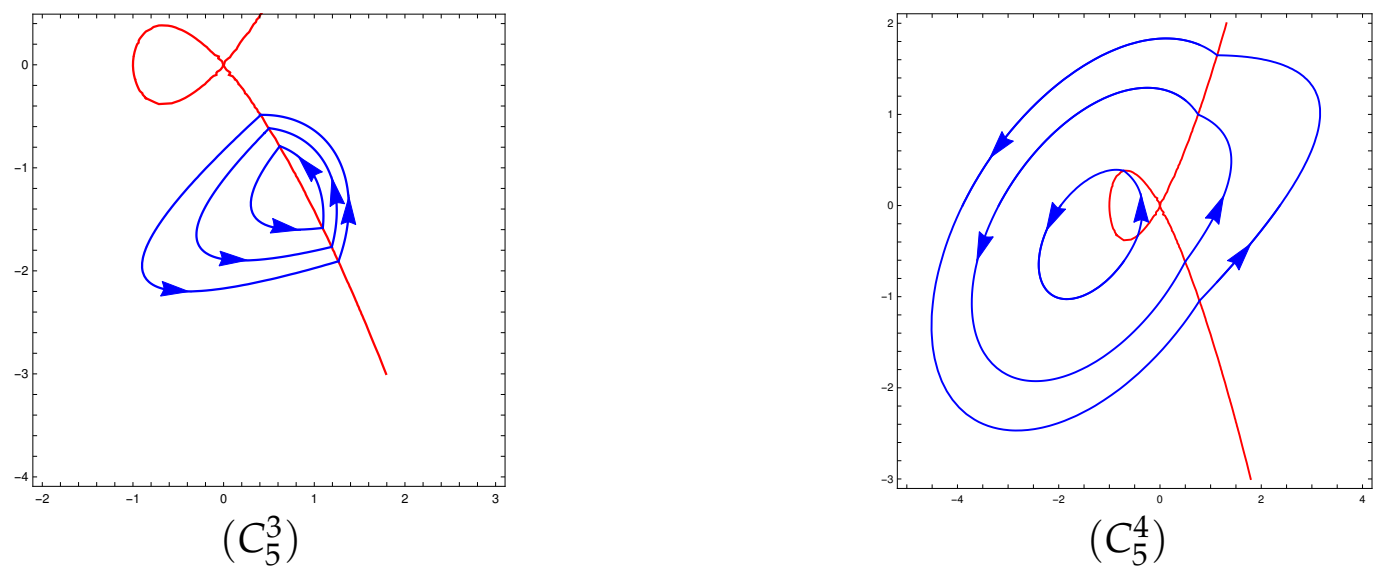

FigurE 11. The three limit cycles of the discontinuous piecewise differential system $\left(C_{5}^{3}\right)$ for $(2.31)-(2.32)$, and $\left(C_{5}^{4}\right)$ for $(2.33)-(2.34)$

For that we suppose that these systems have four crossing limit cycles intersecting the curve $y^{2}-x^{3}=0$ in the points $\left(x_{i}, y_{i}\right)$ and $\left(z_{i}, w_{i}\right)$ for $i=1, \ldots, 4$, which means that they satisfy the system

$$
\begin{aligned}
& H_{1}\left(x_{i}, y_{i}\right)-H_{1}\left(z_{i}, w_{i}\right)=0, \\
& H_{2}\left(x_{i}, y_{i}\right)-H_{2}\left(z_{i}, w_{i}\right)=0, \\
& y_{i}^{2}-x_{i}^{3}=0, \\
& w_{i}^{2}-z_{i}^{3}=0,
\end{aligned}
$$

such points can take the form $A_{i}=\left(r_{i}^{2}, r_{i}^{3}\right)$ and $B_{i}=\left(s_{i}^{2}, s_{i}^{3}\right)$. 

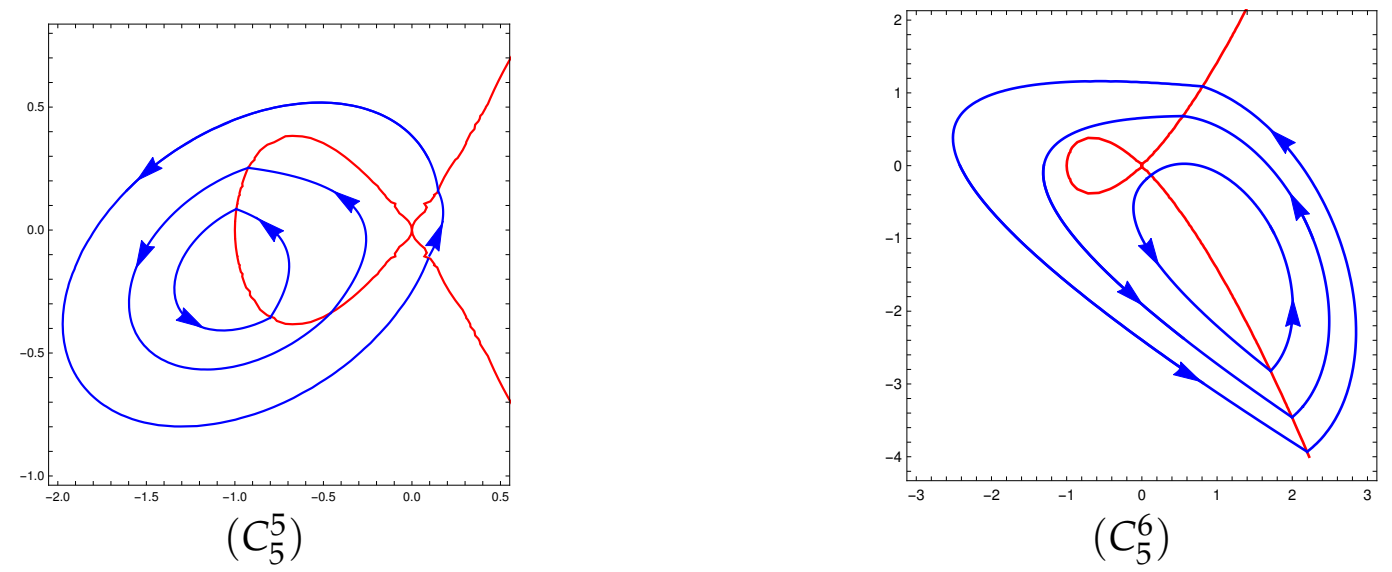

Figure 12. The three limit cycles of the discontinuous piecewise differential system $\left(C_{5}^{5}\right)$ for $(2.35)-(2.36)$, and $\left(C_{5}^{6}\right)$ for $(2.37)-(2.38)$

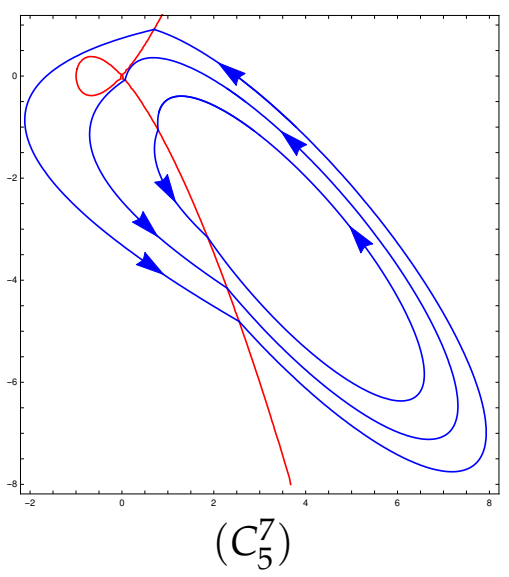

FIGURE 13. The three limit cycles of the discontinuous piecewise differential systems (2.39)-(2.40)

Taking in consideration that these points verify the first two equations of system (2.3), then by solving the first and the second equations when $i=1$, for the parameters $\sigma$ and $d$ we get

$$
\begin{aligned}
\sigma= & \frac{1}{2\left(r_{1}+s_{1}\right)}\left(b_{1} r_{1}^{5}+b_{1} r_{1}^{4} s_{1}+b_{1} r_{1}^{3} s_{1}^{2}+b_{1} r_{1}^{2} s_{1}^{3}+b_{1} r_{1} s_{1}^{4}+b_{1} s_{1}^{5}-2 b_{1} r_{1}^{4} \lambda-2 b_{1} r_{1}^{3} s_{1} \lambda\right. \\
& -2 b_{1} r_{1}^{2} s_{1}^{2} \lambda-2 b_{1} r_{1} s_{1}^{3} \lambda-2 b_{1} s_{1}^{4} \lambda+b_{1} r_{1}^{3} \lambda^{2}+b_{1} r_{1}^{2} s_{1} \lambda^{2}+b_{1} r_{1} s_{1}^{2} \lambda^{2}+b_{1} s_{1}^{3} \lambda^{2}+2 r_{1}^{2} \mu \\
& \left.+2 r_{1} s_{1} \mu+2 s_{1}^{2} \mu\right), \\
d= & \frac{1}{8\left(r_{1}^{2}+r_{1} s_{1}+s_{1}^{2}\right)}\left(8 c\left(r_{1}+s_{1}\right)+4\left(r_{1}+b_{2} r_{1}^{2}+s_{1}+b_{2} r_{1} s_{1}+b_{2} s_{1}^{2}\right)\left(r_{1}^{2}+b_{2} r_{1}^{3}+s_{1}^{2}\right.\right. \\
& \left.\left.+b_{2} s_{1}^{3}\right)+\left(r_{1}+s_{1}\right)\left(r_{1}^{2}-r_{1} s_{1}+s_{1}^{2}\right)\left(r_{1}^{2}+r_{1} s_{1}+s_{1}^{2}\right) \omega^{2}\right) .
\end{aligned}
$$

Since the points $A_{2}=\left(r_{2}^{2}, r_{2}^{3}\right)$ and $B_{2}=\left(s_{2}^{2}, s_{2}^{3}\right)$ also verify system (2.3), we obtain the following expressions of the parameters $\mu=F_{1} / G_{1}$ and $c=F_{2} / G_{2}$ where 


$$
\begin{aligned}
F_{1}= & -\left(b _ { 1 } ( r _ { 2 } - s _ { 2 } ) \left(\left(r_{1}+s_{1}\right)\left(r_{2}+s_{2}\right)\left(r_{1}^{4}-r_{2}^{4}+r_{1}^{2} s_{1}^{2}+s_{1}^{4}-r_{2}^{2} s_{2}^{2}-s_{2}^{4}\right)-2\left(r _ { 2 } \left(r_{1}^{4}\right.\right.\right.\right. \\
& \left.+r_{1}^{3} s_{1}-r_{2}^{3} s_{1}+r_{1}^{2} s_{1}^{2}+s_{1}^{4}+r_{1}\left(-r_{2}^{3}+s_{1}^{3}\right)\right)+\left(r_{1}^{4}+r_{1}^{3} s_{1}-r_{2}^{3} s_{1}+r_{1}^{2} s_{1}^{2}+s_{1}^{4}\right. \\
& \left.\left.+r_{1}\left(-r_{2}^{3}+s_{1}^{3}\right)\right) s_{2}-r_{2}^{2}\left(r_{1}+s_{1}\right) s_{2}^{2}-r_{2}\left(r_{1}+s_{1}\right) s_{2}^{3}-\left(r_{1}+s_{1}\right) s_{2}^{4}\right) \lambda+\left(r_{1}+s_{1}\right) \\
& \left.\left(r_{2}+s_{2}\right)\left(r_{1}^{2}-r_{2}^{2}+s_{1}^{2}-s_{2}^{2}\right) \lambda^{2}\right), \\
G_{1}= & 2\left(r_{2}^{2}\left(r_{1}^{2}+r_{1}\left(-r_{2}+s_{1}\right)+s_{1}\left(-r_{2}+s_{1}\right)\right)-\left(r_{1}^{2}+r_{1} s_{1}+s_{1}^{2}\right) s_{2}^{2}+\left(r_{1}+s_{1}\right) s_{2}^{3}\right),
\end{aligned}
$$

and

$$
\begin{aligned}
F_{2}= & r_{1}^{5}\left(r_{2}^{3}-s_{2}^{3}\right)\left(4 b_{2}^{2}+\omega^{2}\right)+r_{1}^{4}\left(r_{2}^{3}-s_{2}^{3}\right)\left(4 b_{2}\left(2+b_{2} s_{1}\right)+s_{1} \omega^{2}\right)+r_{1}^{3}\left(r_{2}^{3}-s_{2}^{3}\right)(4(1 \\
& \left.\left.+b_{2} s_{1}\right)^{2}+s_{1}^{2} \omega^{2}\right)+r_{1}^{2}\left(-4 r_{2}^{4}-8 b_{2} r_{2}^{5}-r_{2}^{6}\left(4 b_{2}^{2}+\omega^{2}\right)+r_{2}^{3} s_{1}\left(4\left(1+b_{2} s_{1}\right)^{2}+s_{1}^{2} \omega^{2}\right)\right. \\
& \left.+s_{2}^{3}\left(-4 s_{1}-8 b_{2} s_{1}^{2}+4 s_{2}\left(1+b_{2} s_{2}\right)^{2}+s_{2}^{3} \omega^{2}-s_{1}^{3}\left(4 b_{2}^{2}+\omega^{2}\right)\right)\right)+r_{1} s_{1}\left(-4 r_{2}^{4}\right. \\
& -8 b_{2} r_{2}^{5}-r_{2}^{6}\left(4 b_{2}^{2}+\omega^{2}\right)+r_{2}^{3} s_{1}\left(4\left(1+b_{2} s_{1}\right)^{2}+s_{1}^{2} \omega^{2}\right)+s_{2}^{3}\left(-4 s_{1}-8 b_{2} s_{1}^{2}+4 s_{2}\right. \\
& \left.\left.\left(1+b_{2} s_{2}\right)^{2}+s_{2}^{3} \omega^{2}-s_{1}^{3}\left(4 b_{2}^{2}+\omega^{2}\right)\right)\right)+s_{1}^{2}\left(-4 r_{2}^{4}-8 b_{2} r_{2}^{5}-r_{2}^{6}\left(4 b_{2}^{2}+\omega^{2}\right)+r_{2}^{3} s_{1}\right. \\
& \left.\left(4\left(1+b_{2} s_{1}\right)^{2}+s_{1}^{2} \omega^{2}\right)+s_{2}^{3}\left(-4 s_{1}-8 b_{2} s_{1}^{2}+4 s_{2}\left(1+b_{2} s_{2}\right)^{2}+s_{2}^{3} \omega^{2}-s_{1}^{3}\left(4 b_{2}^{2}+\omega^{2}\right)\right)\right), \\
G_{2}= & 8\left(r_{2}^{2}\left(r_{1}^{2}+r_{1}\left(-r_{2}+s_{1}\right)+s_{1}\left(-r_{2}+s_{1}\right)\right)-\left(r_{1}^{2}+r_{1} s_{1}+s_{1}^{2}\right) s_{2}^{2}+\left(r_{1}+s_{1}\right) s_{2}^{3}\right) .
\end{aligned}
$$

Likewise the points $A_{3}=\left(r_{3}^{2}, r_{3}^{3}\right)$ and $B_{3}=\left(s_{3}^{2}, s_{3}^{3}\right)$ satisfy system (2.3), then from the first equation we obtain two values of $\lambda$ named $\lambda^{(1)}$ and $\lambda^{(2)}$ where $\lambda^{(1,2)}=\left(R_{1} \pm(1 / 2) \sqrt{R_{2}}\right) / R_{3}$, and from the second equation we obtain $\omega=-2 \sqrt{D_{1} / D_{2}}$. The values of $R_{1}, R_{2}, R_{3}, D_{1}$ and $D_{2}$ are given in the appendix.

Since the points $A_{4}=\left(r_{4}^{2}, r_{4}^{3}\right)$ and $B_{4}=\left(s_{4}^{2}, s_{4}^{3}\right)$ satisfy system (2.3), then we obtain $b_{1}=0$ from the first equation. This is a contradiction to the assumption.

In the final, we proved that the maximum number of limit cycles for systems of family $\mathcal{F}_{1}$ separated by the irreducible cubic curve $c_{3}=0$ is at most three.

Now we shall provide differential systems of family $\mathcal{F}_{1}$ separated by the cubic $c_{k}=0$ with three limit cycles for $k=1, \ldots, 5$.

We will explain the method for constructing an example of three crossing limit cycles intersecting the curve $c_{1}=0$ in two points, and by a similar way we build examples of three crossing limit cycles intersecting the curve $c_{k}=0$ in two points, when $k \in\{2,3,4,5\}$.

Example 1. Three limit cycles when the cubic of separation is $c_{1}=0$. To construct three crossing limit cycles intersecting the curve $c_{1}=0$ in two points $(\alpha, \beta)$ and $(\gamma, \delta)$, these points must satisfy system of equations

$$
\begin{aligned}
& e_{1}=H_{1}(\alpha, \beta)-H_{1}(\gamma, \delta)=0, \\
& e_{2}=H_{2}(\alpha, \beta)-H_{2}(\gamma, \delta)=0, \\
& c_{i}(\alpha, \beta)=0, \quad c_{i}(\gamma, \delta)=0,
\end{aligned}
$$

when $i=1$. Then we suppose the existence of three real solutions of (2.4) given by

$$
\begin{aligned}
& \left(\alpha_{1}, \beta_{1}, \gamma_{1}, \delta_{1}\right)=(0.196374 . .,-0.492452 . ., 0.16032 . ., 0.436053 . .), \\
& \left(\alpha_{2}, \beta_{2}, \gamma_{2}, \delta_{2}\right)=(0.493859 . .,-0.926394 . ., 0.509449 . ., 0.94932 . .), \\
& \left(\alpha_{3}, \beta_{3}, \gamma_{3}, \delta_{3}\right)=(0.671593 . .,-1.19396 . ., 0.7124 . ., 1.25756 . .) .
\end{aligned}
$$

So, by using equations $e_{1}$ we obtain the values of the three parameters $\lambda, \gamma$ and $\sigma$ in function of $b_{1}$, after that we fixe $b_{1}=5$ we obtain in the region $R_{1}=\left\{(x, y): y^{2}-x\left(x^{2}+x+1\right) \geq 0\right\}$, 
the linear Hamiltonian system

$$
(\dot{x}, \dot{y})=\left(-\frac{x}{2}+5 y+\frac{1}{5},-\frac{x}{20}+\frac{y}{2}+\frac{4}{5}\right),
$$

with its Hamiltonian function $H_{1}(x, y)=-\frac{x^{2}}{40}+\frac{x y}{2}+\frac{4 x}{5}-\frac{5 y^{2}}{2}-\frac{y}{5}$.

Now by using equations $e_{2}$ we obtain the values of the three parameters $b_{2}, c$ and $d$ in function of $\omega$, after that we fixe $\omega=3$. Therefore we obtain in the region $R_{2}=\{(x, y)$ : $\left.y^{2}-x\left(x^{2}+x+1\right) \leq 0\right\}$ the linear differential center

$$
(\dot{x}, \dot{y})=(0.271713 . . x-2.32383 . . y-0.10829 . ., x-0.271713 . . y-0.332792 . .) \text {. }
$$

This differential center has the first integral $H_{2}(x, y)=4(x-0.271713 . . y)^{2}+8(0.10829 . . y-$ 0.332792..x $+9 y^{2}$.

Then the discontinuous piecewise differential system (2.5)-(2.6) has exactly three limit cycles, see Figure 3.

Example 2. Three limit cycles when the cubic of separation is $c_{3}=0$. In the region $R_{1}=\left\{(x, y): y^{2}-x^{3} \geq 0\right\}$ we consider the Hamiltonian system

$$
(\dot{x}, \dot{y})=\left(-\frac{x}{2}+5 y+\frac{1}{5},-\frac{x}{20}+\frac{y}{2}+\frac{4}{5}\right) \text {. }
$$

It has the Hamiltonian function $H_{1}(x, y)=-\frac{x^{2}}{40}+\frac{x y}{2}+\frac{4 x}{5}-\frac{5 y^{2}}{2}-\frac{y}{5}$.

In the region $R_{2}=\left\{(x, y): y^{2}-x^{3} \leq 0\right\}$ we consider the linear differential center

$$
(\dot{x}, \dot{y})=(-0.0813117 . . x-1.00661 . . y+0.56076 . ., x+0.0813117 . . y+8.22904 . .) \text {. }
$$

This differential center has the first integral $H_{2}(x, y)=4(x+0.0813117 . . y)^{2}+8(8.22904 . . x-$ $0.56076 . . y)+4 y^{2}$.

The discontinuous piecewise differential system (2.7)-(2.8) has exactly three limit cycles, because the system of equations (2.4) when $i=3$ has only three real solutions

$$
\begin{aligned}
& \left(\alpha_{1}, \beta_{1}, \gamma_{1}, \delta_{1}\right)=(0.700707 . .,-0.58655 . ., 0.765078 . ., 0.669204 . .), \\
& \left(\alpha_{2}, \beta_{2}, \gamma_{2}, \delta_{2}\right)=(0.969795 . .,-0.955036 . ., 1.06038 . ., 1.09192 . .), \\
& \left(\alpha_{3}, \beta_{3}, \gamma_{3}, \delta_{3}\right)=(1.13263 . .,-1.2054 . ., 1.23647 . ., 1.37492 . .),
\end{aligned}
$$

see Figure 4.

Example 3. Three limit cycles when the cubic of separation is $c_{4}=0$. We consider the Hamiltonian system

$$
(\dot{x}, \dot{y})=\left(\frac{2 x}{5}-4 y+\frac{2}{5}, \frac{x}{25}-\frac{2 y}{5}+\frac{1}{2}\right),
$$

in the region $R_{1}=\left\{(x, y): y^{2}-x^{2}(x-1) \leq 0\right\}$. This Hamiltonian system has the Hamiltonian function $H_{1}(x, y)=\frac{x^{2}}{50}-\frac{2 x y}{5}+\frac{x}{2}+2 y^{2}-\frac{2 y}{5}$.

In the region $R_{2}=\left\{(x, y): y^{2}-x^{2}(x-1) \geq 0\right\}$ we consider the linear center

$$
(\dot{x}, \dot{y})=(0.206345 . . x-2.29258 . . y+0.369992 . ., x-0.206345 . . y-0.332766 . .) \text {, }
$$


which has the first integral $H_{2}(x, y)=4(x-0.206345 . . y)^{2}+8(-0.332766 . . x-0.369992 . . y)+$ $9 y^{2}$.

The discontinuous piecewise differential system (2.9)-(2.10) has exactly three limit cycles, because the system of equations (2.4) when $i=4$ has only three real solutions

$$
\begin{aligned}
& \left(\alpha_{1}, \beta_{1}, \gamma_{1}, \delta_{1}\right)=(1.29278 . .,-0.699517 . ., 1.55103 . ., 1.15135 . .), \\
& \left(\alpha_{2}, \beta_{2}, \gamma_{2}, \delta_{2}\right)=(1.18739 . .,-0.513997 . ., 1.42939 . ., 0.936654 . .), \\
& \left(\alpha_{3}, \beta_{3}, \gamma_{3}, \delta_{3}\right)=(1.05976 . .,-0.259058 . ., 1.25796 . ., 0.638917 . .),
\end{aligned}
$$

see Figure 5.

Seven examples with three limit cycles when the cubic of separation is $c_{2}=0$. We define the following regions associated to the curve $c_{2}=0$.

$$
\begin{aligned}
& R_{1}=\left\{(x, y): y^{2}-x(x-1)(x-3) \geq 0\right\}, \\
& R_{2}=\left\{(x, y): y^{2}-x(x-1)(x-3) \leq 0, x \geq 3\right\}, \\
& R_{3}=\left\{(x, y): y^{2}-x(x-1)(x-3) \leq 0,0 \leq x \leq 1\right\} .
\end{aligned}
$$

Example 4.1. For the first configuration of limit cycles separated by the curve $c_{2}=0$ we consider the Hamiltonian system

$$
(\dot{x}, \dot{y})=\left(\frac{39}{10} x-3 y-\frac{3}{5}, 0.0507 . . x-\frac{39}{10} y+1\right),
$$

in the region $R_{2}$. It has the Hamiltonian function $H_{1}(x, y)=0.02535 . . x^{2}-\frac{39}{10} x y+x+\frac{3 y^{2}}{2}+\frac{3 y}{5}$.

In the region $R_{1}$ we consider the linear diifferential center

$$
(\dot{x}, \dot{y})=(0.138717 . . x-1.01924 . . y-0.161038 . ., x-0.138717 . . y-1.51992 . .),
$$

which has the first integral $H_{2}(x, y)=4(x-0.138717 . . y)^{2}+8(0.161038 . . y-1.51992 . . x)+4 y^{2}$.

The discontinuous piecewise differential system (2.12)-(2.13) has exactly three limit cycles, because the system of equations (2.4) when $i=2$, has only the three real solutions

$$
\begin{aligned}
& \left(\alpha_{1}, \beta_{1}, \gamma_{1}, \delta_{1}\right)=(3.04422 . .,-0.524589 . ., 3.11914 . ., 0.887429 . .), \\
& \left(\alpha_{2}, \beta_{2}, \gamma_{2}, \delta_{2}\right)=(3.1128 . .,-0.861296 . ., 3.21824 . ., 1.24817 . .), \\
& \left(\alpha_{3}, \beta_{3}, \gamma_{3}, \delta_{3}\right)=(3.17852 . .,-1.11182 . ., 3.30364 . ., 1.52013 . .),
\end{aligned}
$$

see $\left(C_{2}^{1}\right)$ of Figure 6 .

Example 4.2. For the second configuration we consider the Hamiltonian system

$$
(\dot{x}, \dot{y})=\left(1 . x-5 y-\frac{3}{10}, \frac{1}{5} x-y-2\right),
$$

in the region $R_{1}$. It has the Hamiltonian function $H_{1}(x, y)=\frac{1}{10} x^{2}-x y-2 x+\frac{5 y^{2}}{2}+\frac{3}{10} y$. Now we consider the second Hamiltonian system

$$
(\dot{x}, \dot{y})=(0.214743 . . x-1.04611 y+0.0404675 . ., x-0.214743 . . y-2.71521 . .),
$$

in the region $R_{2}$. This system has the first integral $H_{2}(x, y)=4(x-0.311552 . . y)^{2}+8(0.286927 . . y-$ 3.12887..x) $+9 y^{2}$. 
The discontinuous piecewise differential sysem (2.14)-(2.15) has exactly three limit cycles, because the system of equations (2.4) when $i=2$, has only the three real solutions

$$
\begin{aligned}
& \left(\alpha_{1}, \beta_{1}, \gamma_{1}, \delta_{1}\right)=(3.00992 . .,-0.244971 . ., 3.30407 . ., 1.52144 . .), \\
& \left(\alpha_{2}, \beta_{2}, \gamma_{2}, \delta_{2}\right)=(3.02738 . .,-0.409955 . ., 3.36189 . ., 1.69517 . .), \\
& \left(\alpha_{3}, \beta_{3}, \gamma_{3}, \delta_{3}\right)=(3.04843 . .,-0.549942 . ., 3.41294 . ., 1.84408 . .),
\end{aligned}
$$

see $\left(C_{2}^{2}\right)$ of Figure 6 .

Example 4.3. To obtain the third configuration we consider in the region $R_{1}$ the Hamiltonian system

$$
(\dot{x}, \dot{y})=\left(0.63 x-3 y-\frac{1}{5},-\frac{23 x}{1000}-\frac{23 y}{100}+1\right),
$$

which has the Hamiltonian function $H_{1}(x, y)=0.06615 x^{2}-0.63 x y-2.4 x+\frac{3 y^{2}}{2}+0.2 y$. In the region $R_{2}$ we consider the linear center

$$
(\dot{x}, \dot{y})=(0.222578 x-1.04954 y+0.0489144 . ., x-0.222578 . . y-3.2101 . .) .
$$

This differential system has the first integral $H_{2}(x, y)=4(x-0.222578 . . y)^{2}+8(-3.2101 . . x-$ $0.0489144 . . y)+4 y^{2}$.

The discontinuous piecewise differential system (2.16)-(2.17) has exactly three limit cycles, due to the fact that the system (2.4) has three real solutions when $i=2$. These solutions are

$$
\begin{aligned}
& \left(\alpha_{1}, \beta_{1}, \gamma_{1}, \delta_{1}\right)=(3.20789 . .,-1.21343 . ., 3.74104 . ., 2.7566 . .), \\
& \left(\alpha_{2}, \beta_{2}, \gamma_{2}, \delta_{2}\right)=(3.3699 . .,-1.71878 . ., 3.9416 . ., 3.30416 . .), \\
& \left(\alpha_{3}, \beta_{3}, \gamma_{3}, \delta_{3}\right)=(3.51026 . .,-2.12043 . ., 4.10331 . ., 3.74825 . .),
\end{aligned}
$$

see $\left(C_{2}^{3}\right)$ of Figure 7.

Example 4.4. To obtain the fourth configuration we consider in the region $R_{1}$ the Hamiltonian system

$$
(\dot{x}, \dot{y})=\left(\frac{1}{5} x-y-\frac{1}{5}, 0.04 x-\frac{1}{5} y-2\right),
$$

which has the Hamiltonian function $H_{1}(x, y)=0.02 . x^{2}-\frac{1}{5} x y-2 x+\frac{y^{2}}{2}+\frac{1}{5} y$.

In the region $R_{2}$ we consider the linear center

$$
\begin{aligned}
(\dot{x}, \dot{y})= & (0.0193278 . . x-0.0628736 . . y+0.0594448 . ., x-0.0193278 . . y \\
& -2.86499 . .) .
\end{aligned}
$$

This differential system has the first integral $H_{2}(x, y)=4(x-0.0193278 . . y)^{2}+8(-2.86499 . . x-$ 0.0594448..y) $+\frac{y^{2}}{4}$.

The discontinuous piecewise differential system (2.18)-(2.19) has exactly three limit cycles, because the system (2.4) has three real solutions when $i=2$, which are

$$
\begin{aligned}
& \left(\alpha_{1}, \beta_{1}, \gamma_{1}, \delta_{1}\right)=(3.33264 . .,-1.60807 . ., 3.86848 . ., 3.1044 . .), \\
& \left(\alpha_{2}, \beta_{2}, \gamma_{2}, \delta_{2}\right)=(3.71366 . .,-2.68179 . ., 4.26489 . ., 4.19675 . .), \\
& \left(\alpha_{3}, \beta_{3}, \gamma_{3}, \delta_{3}\right)=(3.97997 . .,-3.40919 . ., 4.54158 . ., 4.9795 . .),
\end{aligned}
$$


see $\left(C_{2}^{4}\right)$ of Figure 7 .

Example 4.5. For the fifth configuration we consider in the region $R_{1}$ the Hamiltonian system

$$
(\dot{x}, \dot{y})=\left(-\frac{14 x}{5}-7 y+\frac{163}{100}, \frac{28 x}{25}+\frac{14 y}{5}+\frac{2}{5}\right),
$$

which has the Hamiltonian function $H_{1}(x, y)=\frac{14 x^{2}}{25}+\frac{14 x y}{5}+\frac{2 x}{5}+\frac{7 y^{2}}{2}-\frac{163 y}{100}$. In the region $R_{3}$ we consider the linear center

$$
\begin{aligned}
(\dot{x}, \dot{y})= & (0.338865 . . x-0.142607 . . y-0.170936 . ., 0.338865 . . x \\
& -0.142607 . . y-0.170936 . .) .
\end{aligned}
$$

This differential system has the first integral $H_{2}(x, y)=4(x-0.338865 . . y)^{2}+8(0.170936 . . y-$ 0.0452618..x $+\frac{y^{2}}{9}$.

When $i=2$ in the system of equations (2.4) the discontinuous piecewise differential system (2.20)-(2.21) has exactly three limit cycles intersecting the cubic curve $c_{2}=0$ in the points

$$
\begin{aligned}
& \left(\alpha_{1}, \beta_{1}, \gamma_{1}, \delta_{1}\right)=(0.748414 . ., 0.651116 . ., 0.605489 . .,-0.756295 . .), \\
& \left(\alpha_{2}, \beta_{2}, \gamma_{2}, \delta_{2}\right)=(0.911161 . ., 0.4112 . ., 0.747078 . .,-0.652453 . .), \\
& \left(\alpha_{3}, \beta_{3}, \gamma_{3}, \delta_{3}\right)=(0.988069 . ., 0.154006 . ., 0.87431 . .,-0.483319 . .),
\end{aligned}
$$

see $\left(C_{2}^{5}\right)$ of Figure 8 .

Example 4.6. Now we give un example for the sixth configuration. We consider in the region $R_{1}$ the Hamiltonian system

$$
(\dot{x}, \dot{y})=(62.8408 . . x+3 y+11.8867 . .,-1316.32 . . x-62.8408 . . y+2292.58 . .),
$$

with its Hamiltonian function $H_{1}(x, y)=-658.162 . . x^{2}-62.8408 . . x y+2292.58 . . x-\frac{3 y^{2}}{2}-11.8867 . . y$. In the region $R_{2} \cup R_{3}$ we consider the linear center

$$
(\dot{x}, \dot{y})=(-0.4 x-2.12 . . y+0.2 . ., x+0.4 y-1.58 . .) .
$$

This differential system has the first integral $H_{2}(x, y)=4 x^{2}+3.2 . . x y-12.64 x+8.48 . . y^{2}-1.6 . . y$.

For this configuration the system (2.4) has three real solutions when $i=2$. Hence, the discontinuous piecewise differential system (2.22)-(2.23) has exactly three limit cycles given by

$$
\begin{aligned}
& \left(\alpha_{1}, \beta_{1}, \gamma_{1}, \delta_{1}\right)=(0.953136 . ., 0.302372 . ., 0.905745 . .,-0.422833 . .), \\
& \left(\alpha_{2}, \beta_{2}, \gamma_{2}, \delta_{2}\right)=(0.788975 . ., 0.60673 . ., 0.73033 . .,-0.668586 . .), \\
& \left(\alpha_{3}, \beta_{3}, \gamma_{3}, \delta_{3}\right)=(3.02633 . ., 0.401853 . ., 3.21618 . .,-1.24131 . .),
\end{aligned}
$$

see $\left(C_{2}^{6}\right)$ of Figure 8 .

Example 4.7. Finaly for the seventh configuration we consider in the region $R_{1}$ the Hamiltonian system

$$
(\dot{x}, \dot{y})=(98.4926 . . x+3 y+225.612 . . .,-3233.6 . . x-98.4926 . . y+7255.43 . .),
$$


with its Hamiltonian function $H_{1}(x, y)=-1616.8 . . x^{2}-98.4926 . . x y+7255.43 . . x-\frac{3 y^{2}}{2}-225.612 . . y$.

In the region $R_{2} \cup R_{3}$ we consider the linear center

$$
(\dot{x}, \dot{y})=\left(-\frac{2 x}{5}-\frac{29 y}{25}+\frac{1}{5}, x+\frac{2 y}{5}-\frac{79}{50}\right) .
$$

This differential system has the first integral $H_{2}(x, y)=4 x^{2}+3.2 . . x y-12.64 . . x+4.64 . . y^{2}-$ 1.6..y.

The system of equations (2.4) has three real solutions when $i=2$. So, the discontinuous piecewise differential system (2.24)-(2.25) has exactly three limit cycles intersecting the cubic curve $c_{2}=0$ in the points

$$
\begin{aligned}
& \left(\alpha_{1}, \beta_{1}, \gamma_{1}, \delta_{1}\right)=(0.78786 . ., 0.608054 . ., 0.707741 . .,-0.688578 . .), \\
& \left(\alpha_{2}, \beta_{2}, \gamma_{2}, \delta_{2}\right)=(3.02845 . ., 0.418065 . ., 3.43049 . .,-1.89457 . .), \\
& \left(\alpha_{3}, \beta_{3}, \gamma_{3}, \delta_{3}\right)=(3.07065 . ., 0.670216 . ., 3.54067 . .,-2.20536 . .),
\end{aligned}
$$

see $\left(C_{2}^{7}\right)$ of Figure 9 .

Seven examples with three limit cycles when the cubic of separation is $c_{5}=0$. We define the following three regions associated to the curve $c_{5}=0$

$$
\begin{aligned}
& R_{1}=\left\{(x, y): y^{2}-x^{2}(x+1) \leq 0, x \geq 0\right\} \\
& R_{2}=\left\{(x, y): y^{2}-x^{2}(x+1) \geq 0\right\}, \\
& R_{3}=\left\{(x, y): y^{2}-x^{2}(x+1) \leq 0,-1 \leq x \leq 0\right\} .
\end{aligned}
$$

Example 5.1. For the first configuration of the class $C_{5}$ and in the region $R_{1}$ we consider the Hamiltonian system

$$
(\dot{x}, \dot{y})=\left(\frac{9 x}{10}-3 y+\frac{1}{5}, \frac{27 x}{100}-\frac{9 y}{10}+\frac{6}{5}\right),
$$

which has the Hamiltonian function $H_{1}(x, y)=\frac{27 x^{2}}{200}-\frac{9 x y}{10}+\frac{6 x}{5}+\frac{3 y^{2}}{2}-\frac{y}{5}$.

In the region $R_{2}$ we consider the linear differential center

$$
(\dot{x}, \dot{y})=(0.148466 . . x-0.744542 . . y+0.46566 . ., x-0.148466 . . y+2.05075 . .) \text {. }
$$

Its corresponding first integral is $H_{2}(x, y)=4(x-0.148466 . . y)^{2}+8(2.05075 . . x-0.46566 . . y)+$ $\frac{289 y^{2}}{100}$.

The discontinuous piecewise differential system (2.27)-(2.28) has exactly three limit cycles, because the system of equations (2.4) when $i=5$, has the three real solutions

$$
\begin{aligned}
& \left(\alpha_{1}, \beta_{1}, \gamma_{1}, \delta_{1}\right)=(0.482359 . .,-0.587282 . ., 0.727878 . ., 0.956787 . .), \\
& \left(\alpha_{2}, \beta_{2}, \gamma_{2}, \delta_{2}\right)=(0.644665 . .,-0.826748 . ., 0.94961 . ., 1.32593 . .), \\
& \left(\alpha_{3}, \beta_{3}, \gamma_{3}, \delta_{3}\right)=(0.770799 . .,-1.02571 . ., 1.11523 . ., 1.62197 . .),
\end{aligned}
$$

see $\left(C_{5}^{1}\right)$ of Figure 10. 
Example 5.2. For the second configuration of the class $C_{5}$ and in the region $R_{1}$ we consider the Hamiltonian system

$$
(\dot{x}, \dot{y})=\left(-\frac{42 x}{25}-8 y+1, \frac{441 x}{1250}+\frac{42 y}{25}+\frac{12}{5}\right),
$$

with its Hamiltonian function $H_{1}(x, y)=\frac{441 x^{2}}{2500}+\frac{42 x y}{25}+\frac{12 x}{5}+4 y^{2}-y$.

Now we consider the linear center

$$
\begin{aligned}
(\dot{x}, \dot{y})= & (-0.680215 . . x-2.02519 . . y-0.290646 . ., x+0.680215 . . y \\
& +0.771302 . .)
\end{aligned}
$$

in the region $R_{2}$. This differential system has the first integral $H_{2}(x, y)=4(x+0.680215 . . y)^{2}+$ $8(0.771302 . . x+0.290646 . . y)+6.25 . . y^{2}$.

The discontinuous piecewise differential system (2.29)-(2.30) has exactly three limit cycles, because the system of equations (2.4) when $i=5$, has the three real solutions

$$
\begin{aligned}
& \left(\alpha_{1}, \beta_{1}, \gamma_{1}, \delta_{1}\right)=(-0.999059 . ., 0.0306461 . .,-0.857795 . ., 0.323475 . .), \\
& \left(\alpha_{2}, \beta_{2}, \gamma_{2}, \delta_{2}\right)=(-0.969398 . .,-0.16958 . .,-0.614006 . ., 0.381472 . .), \\
& \left(\alpha_{3}, \beta_{3}, \gamma_{3}, \delta_{3}\right)=(-0.888264 . .,-0.296919 . .,-0.329658 . ., 0.269906 . .),
\end{aligned}
$$

see $\left(C_{5}^{2}\right)$ of Figure 10.

Example 5.3. To obtain the third configuration of the class $C_{5}$ and in the region $R_{2}$ we consider the Hamiltonian system

$$
(\dot{x}, \dot{y})=(2(x-2 y-3),-4+x-2 y),
$$

which has the Hamiltonian function $H_{1}(x, y)=\frac{x^{2}}{2}-2 x y-4 x+2 y^{2}+6 y$. In the region $R_{1}$ we consider the linear center

$$
(\dot{x}, \dot{y})=(0.371399 . . x-1.13794 . . y-2.0777 . ., x-0.371399 . . y-0.612049 . .),
$$

with its first integral $H_{2}(x, y)=4(x-0.371399 y)^{2}+8(2.0777 y-0.612049 x)+4 y^{2}$.

In this case we have also three limit cycles, due to the fact that the system of equations (2.4) when $i=5$ has the three real solutions. Hence, the discontinuous piecewise differential system (2.31)-(2.32) has exactly three limit cycles intersecting the curve $c_{5}=0$ in the points

$$
\begin{aligned}
& \left(\alpha_{1}, \beta_{1}, \gamma_{1}, \delta_{1}\right)=(1.26688 . .,-1.90744 . ., 0.409461 . .,-0.486115 . .), \\
& \left(\alpha_{2}, \beta_{2}, \gamma_{2}, \delta_{2}\right)=(1.19339 . .,-1.76742 . ., 0.502225 . .,-0.615553 . .), \\
& \left(\alpha_{3}, \beta_{3}, \gamma_{3}, \delta_{3}\right)=(1.09399 . .,-1.58308 . ., 0.620329 . .,-0.78963 . .),
\end{aligned}
$$

see $\left(C_{5}^{3}\right)$ of Figure 11.

Example 5.4. To get the fourth configuration of the class $C_{5}$ and in the region $R_{1} \cup R_{3}$ we consider the Hamiltonian system

$$
(\dot{x}, \dot{y})=\left(\frac{9 x}{10}-3 y+\frac{1}{5}, \frac{27 x}{100}-\frac{9 y}{10}+\frac{6}{5}\right),
$$


which has the Hamiltonian function $H_{1}(x, y)=\frac{27 x^{2}}{200}-\frac{9 x y}{10}+\frac{6 x}{5}+\frac{3 y^{2}}{2}-\frac{y}{5}$.

Now we consider the linear center

$$
(\dot{x}, \dot{y})=(0.685337 . . x-2.15969 . . y+0.24115 . ., x-0.685337 . . y+1.13465 . .),
$$

in the region $R_{2}$. This differential system has the first integral $H_{2}(x, y)=4(x-0.685337 . . y)^{2}+$ $8(1.13465 . . x-0.24115 . . y)+\frac{169 y^{2}}{25}$.

The discontinuous piecewise differential system (2.33)-(2.34) has exactly three limit cycles, because the system of equations (2.4) when $i=5$, has the three real solutions

$$
\begin{aligned}
& \left(\alpha_{1}, \beta_{1}, \gamma_{1}, \delta_{1}\right)=(-0.436282 . .,-0.327565 . .,-0.713688 . ., 0.381881 . .), \\
& \left(\alpha_{2}, \beta_{2}, \gamma_{2}, \delta_{2}\right)=(0.501048 . .,-0.613871 . ., 0.754011 . ., 0.998606 . .), \\
& \left(\alpha_{3}, \beta_{3}, \gamma_{3}, \delta_{3}\right)=(0.782108 . .,-1.04408 . ., 1.12985 . ., 1.64891 . .),
\end{aligned}
$$

see $\left(C_{5}^{4}\right)$ of Figure 11.

Example 5.5. Forn the fifth configuration of the class $C_{5}$ and in the region $R_{1} \cup R_{3}$ we consider the Hamiltonian system

$$
(\dot{x}, \dot{y})=\left(\frac{1}{200}(367 x-1468 y+40), \frac{367 x}{800}-\frac{367 y}{200}+\frac{6}{5}\right),
$$

which has the Hamiltonian function $H_{1}(x, y)=0.229375 . . x^{2}-1.835 . . x y+\frac{6 x}{5}+3.67 . . y^{2}-\frac{y}{5}$. Now we consider the linear center

$$
(\dot{x}, \dot{y})=(0.591399 . . x-2.59975 . . y+0.172769 . ., x-0.591399 . . y+0.826843 . .),
$$

in the region $R_{2}$. This differential system has the first integral $H_{2}(x, y)=4(x-0.591399 . . y)^{2}+$ $8(0.826843 . . x-0.172769 . . y)+9 y^{2}$.

The system of equations (2.4) when $i=5$ has three real solutions, which means that the discontinuous piecewise differential system (2.35)-(2.36) has exactly three limit cycles intesecting the curve $c_{5}=0$ in the following points

$$
\begin{aligned}
& \left(\alpha_{1}, \beta_{1}, \gamma_{1}, \delta_{1}\right)=(-0.801385 . .,-0.357147 . .,-0.992606 . ., 0.0853535 . .), \\
& \left(\alpha_{2}, \beta_{2}, \gamma_{2}, \delta_{2}\right)=(-0.457786 . .,-0.337092 . .,-0.925215 . ., 0.253018 . .), \\
& \left(\alpha_{3}, \beta_{3}, \gamma_{3}, \delta_{3}\right)=(0.0989294 . .,-0.103708 . ., 0.147927 . ., 0.158491 . .),
\end{aligned}
$$

see $\left(C_{5}^{5}\right)$ of Figure 12.

Example 5.6. Now we give the limit cycles of the sixth configuration of the class $C_{5}$. In the region $R_{1}$ we consider the Hamiltonian system

$$
(\dot{x}, \dot{y})=(-5-3.2 x-8 y,-3+1.28 x+3.2 y),
$$

which has the Hamiltonian function $H_{1}(x, y)=0.64 x^{2}+3.2 x y-3 x+4 y^{2}+5 y$. Now we consider in the region $R_{2}$ the linear center

$$
\begin{aligned}
(\dot{x}, \dot{y})= & (-0.213131 . . x-0.535425 . . y-0.615264 . ., x+0.213131 . . y \\
& -0.568363 . .)
\end{aligned}
$$


with its first integral $H_{2}(x, y)=4(x+0.213131 . . y)^{2}+8(0.615264 . . y-0.568363 . . x)+1.96 . . y^{2}$.

The discontinuous piecewise differential system (2.37)-(2.38) has exactly three limit cycles, because the system of equations (2.4) when $i=5$, has the three real solutions

$$
\begin{aligned}
& \left(\alpha_{1}, \beta_{1}, \gamma_{1}, \delta_{1}\right)=(2.19804 . .,-3.93077 . ., 0.809528 . ., 1.08897 . .), \\
& \left(\alpha_{2}, \beta_{2}, \gamma_{2}, \delta_{2}\right)=(1.99735 . .,-3.45798 . ., 0.547303 . ., 0.680793 . .), \\
& \left(\alpha_{3}, \beta_{3}, \gamma_{3}, \delta_{3}\right)=(1.71246 . .,-2.82035 . ., 0.123691 . .,-0.131118 . .),
\end{aligned}
$$

see $\left(C_{5}^{6}\right)$ of Figure 12.

Example 5.7. Finally and for the seventh configuration of the class $C_{5}$ and in the region $R_{1}$ we consider the Hamiltonian system

$$
(\dot{x}, \dot{y})=\left(-\frac{7 x}{5}-7 y-9, \frac{7 x}{25}+\frac{7 y}{5}-5\right)
$$

with its Hamiltonian function $H_{1}(x, y)=\frac{7 x^{2}}{50}+\frac{7 x y}{5}-5 x+\frac{7 y^{2}}{2}+9 y$.

Now we consider the linear center

$$
\begin{aligned}
(\dot{x}, \dot{y})= & (-0.801265 . . x-0.944526 . . y-0.242753 . ., x+0.801265 . . y . . \\
& -0.973031 . .)
\end{aligned}
$$

in the region $R_{2}$. This differential system has the first integral $H_{2}(x, y)=4(x+0.801265 . . y)^{2}+$ $8(0.242753 . . y-0.973031 . . x)+1.21 . . y^{2}$.

Due to the fact that the system of equations (2.4) when $i=5$, has three real solutions, we know that the discontinuous piecewise differential system (2.39)-(2.40) has exactly three limit cycles intersecting the curve $c_{5}=0$ in the points

$$
\begin{aligned}
& \left(\alpha_{1}, \beta_{1}, \gamma_{1}, \delta_{1}\right)=(2.55092 . .,-4.80692 . ., 0.700327 . ., 0.913203 . .), \\
& \left(\alpha_{2}, \beta_{2}, \gamma_{2}, \delta_{2}\right)=(2.29096 . .,-4.15603 . ., 0.0707197 . .,-0.0731776 . .), \\
& \left(\alpha_{3}, \beta_{3}, \gamma_{3}, \delta_{3}\right)=(1.8659 . .,-3.15879 . ., 0.786808 . .,-1.05174 . .),
\end{aligned}
$$

see $\left(C_{5}^{7}\right)$ of Figure 13.

This completes the proof of Theorem 1.2.

\section{Proof of Theorem 1.3}

To prove the statement $(a)$ of Theorem 1.3 , we are going to prove it for the class $C_{5}$, and by a similar way we get the proof of the statements for the class $C_{2}$.

We consider the three regions $R_{i}$ for $i=1,2,3$ defined in (2.26).

In the region $R_{1}$ we consider the linear differential center

$$
\dot{x}=-\frac{1}{4} y\left(4 b_{2}^{2}+\omega^{2}\right)-b_{2} x+d, \dot{y}=b_{2} y+c+x,
$$

its first integral is $H_{2}(x, y)=4\left(b_{2} y+x\right)^{2}+8(c x-d y)+y^{2} \omega^{2}$. 


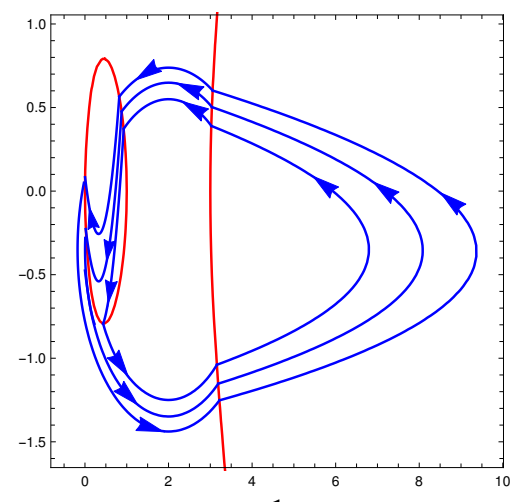

$\left(C_{2}^{1}\right)$

FiguRE 14. The three limit cycles of the discontinuous piecewise differential system $\left(C_{2}^{1}\right)$ for (3.6)-(3.7)

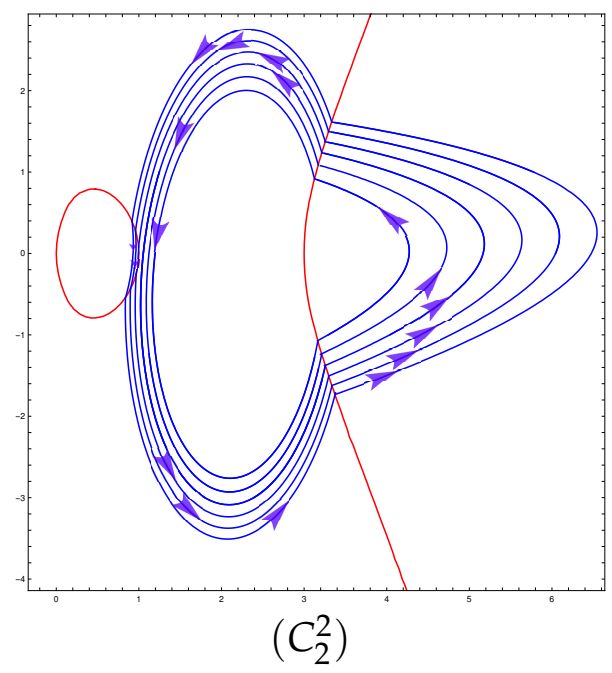

FiguRE 15. The six limit cycles of the discontinuous piecewise differential systems (3.10)-(3.11)

Now, we consider the two Hamiltonian systems

$$
\begin{array}{ll}
\dot{x}=-\lambda_{1} b_{1} x+b_{1} y+\mu_{1}, & \dot{y}=-\lambda_{1}^{2} b_{1} x+\lambda_{1} b_{1} y+\sigma_{1}, \text { in the region } R_{2}, \\
\dot{x}=-\lambda_{3} b_{3} x+b_{3} y+\mu_{3}, & \dot{y}=-\lambda_{3}^{2} b_{3} x+\lambda_{3} b_{3} y+\sigma_{3}, \text { in the region } R_{3},
\end{array}
$$

with $b_{i} \neq 0$ and $\sigma_{i} \neq \lambda_{i} \mu_{i}$, when $i=1,3$. Their corresponding Hamiltonian first integrals are

$$
\begin{aligned}
& H_{1}(x, y)=-\left(\lambda_{1}^{2} b_{1} / 2\right) x^{2}+\lambda_{1} b_{1} x y-\left(b_{1} / 2\right) y^{2}+\sigma_{1} x-\mu_{1} y, \\
& H_{3}(x, y)=-\left(\lambda_{3}^{2} b_{3} / 2\right) x^{2}+\lambda_{3} b_{3} x y-\left(b_{3} / 2\right) y^{2}+\sigma_{3} x-\mu_{3} y .
\end{aligned}
$$

If we suppose that the discontinuous piecewise differential systems (3.1)-(3.2) have three limit cycles which intersect the cubic $c_{5}=0$ in the points $p_{1}^{(i)}=\left(\alpha_{i}^{2}-1, \alpha_{i}\left(\alpha_{i}^{2}-1\right)\right), p_{2}^{(i)}=\left(\beta_{i}^{2}-\right.$ $\left.1, \beta_{i}\left(\beta_{i}^{2}-1\right)\right), p_{3}^{(i)}=\left(\gamma_{i}^{2}-1, \gamma_{i}\left(\gamma_{i}^{2}-1\right)\right)$ and $p_{4}^{(i)}=\left(\delta_{i}^{2}-1, \delta_{i}\left(\delta_{i}^{2}-1\right)\right)$ they must satisfy the 


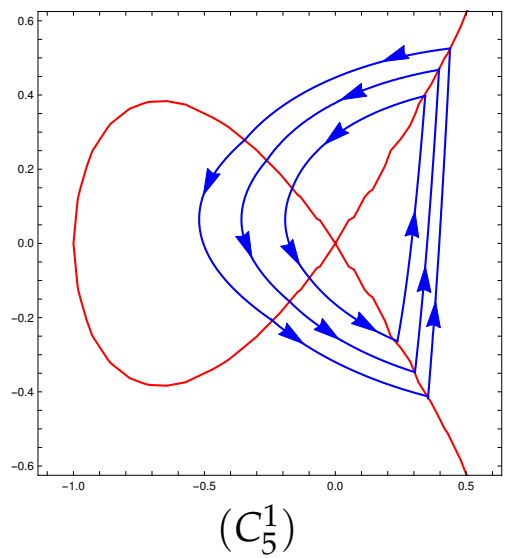

FIGURE 16. The three limit cycles of the discontinuous piecewise differential system $\left(C_{5}^{1}\right)$ for (3.8)-(3.9)

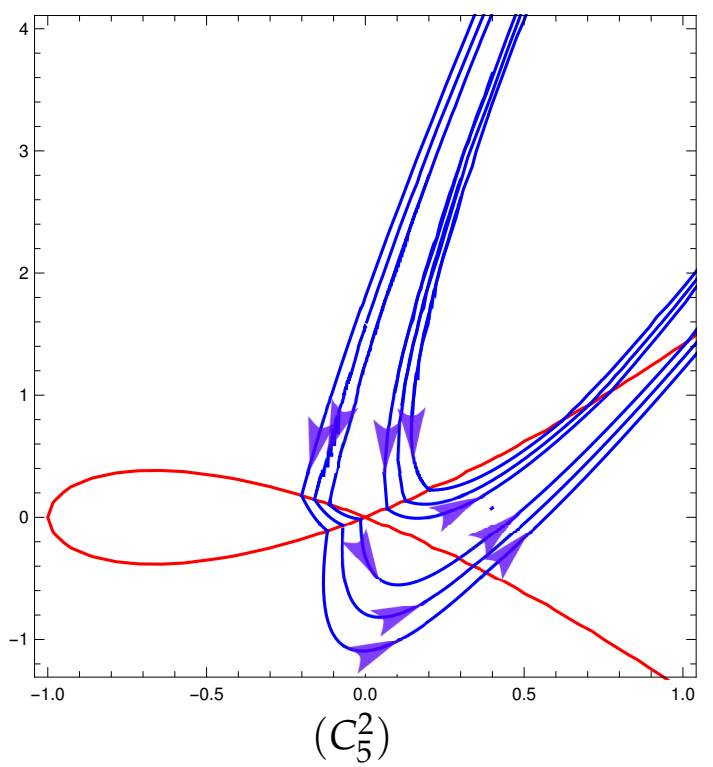

Figure 17. The six limit cycles of the discontinuous piecewise differential system (3.12)-(3.13)

following system

$$
\begin{aligned}
& H_{1}\left(\alpha_{i}^{2}-1, \alpha_{i}\left(\alpha_{i}^{2}-1\right)\right)-H_{1}\left(\beta_{i}^{2}-1, \beta_{i}\left(\beta_{i}^{2}-1\right)\right)=0, \\
& H_{2}\left(\beta_{i}^{2}-1, \beta_{i}\left(\beta_{i}^{2}-1\right)\right)-H_{2}\left(\delta_{i}^{2}-1, \delta_{i}\left(\delta_{i}^{2}-1\right)\right)=0, \\
& H_{2}\left(\alpha_{i}^{2}-1, \alpha_{i}\left(\alpha_{i}^{2}-1\right)\right)-H_{2}\left(\gamma_{i}^{2}-1, \gamma_{i}\left(\gamma_{i}^{2}-1\right)\right)=0, \\
& H_{3}\left(\gamma_{i}^{2}-1, \gamma_{i}\left(\gamma_{i}^{2}-1\right)\right)-H_{3}\left(\delta_{i}^{2}-1, \delta_{i}\left(\delta_{i}^{2}-1\right)\right)=0 .
\end{aligned}
$$

We deal with the first and the last equation. By solving the first one for $i=1,2,3$ we obtain the expression of $\lambda_{1}, \mu_{1}$ and $\sigma_{1}$, and by solving the last equation we get the expression of $\lambda_{3}$, $\mu_{3}$ and $\sigma_{3}$. Now we suppose that system (3.1)-(3.2) has a fourth limit cycle, so system (3.4) satisfied for $i=4$. We solve its first and last equations we get $b_{1}=0$ and $b_{3}=0$, respectively. 
This is a contradiction of the assumption. Thus we prove that the maximum number of limit cycles intersected with the cubic curve $c_{5}=0$ in four points is three.

Example 6. Three limit cycles intersecting the curve $c_{2}=0$ in four points. We consider the regions $R_{i}$ for $i=1,2,3$ defined in (2.11).

We will explain the method for constructing an example of three limit cycles intersecting the curve $c_{2}=0$ in four points. These points must satisfy the system of equations

$$
\begin{aligned}
& \left.e_{1}=H_{1}\left(\alpha_{s}, \beta_{s}\right)\right)-H_{1}\left(\gamma_{s}, \delta_{s}\right)=0, \\
& e_{2}=H_{2}\left(\alpha_{s}, \beta_{s}\right)-H_{2}\left(f_{s}, g_{s}\right)=0, \\
& e_{3}=H_{2}\left(\gamma_{s}, \delta_{s}\right)-H_{2}\left(h_{s}, k_{s}\right)=0 \\
& e_{4}=H_{3}\left(f_{s}, g_{s}\right)-H_{3}\left(h_{s}, k_{s}\right)=0 \\
& c_{i}\left(\alpha_{s}, \beta_{s}\right)=c_{i}\left(\gamma_{s}, \delta_{s}\right)=0 \\
& c_{i}\left(f_{s}, g_{s}\right)=c_{i}\left(h_{s}, k_{s}\right)=0,
\end{aligned}
$$

with $s=1,2,3$ and $i=2$.

In the region $R_{1}$ and by using the software Mathematica we construct the curves

$$
H_{2}(x, y)=4 x^{2}+8\left(\frac{7 y}{5}-2 x\right)+16 y^{2}=3 k-8, \ldots .(I) k \in\{1,2,3\}
$$

Where the level curve corresponding to $k=1$ in $(I)$, intersects the cubic $c_{2}=0$, in $\left(\alpha_{1}, \beta_{1}\right)=$ (3.02482.., 0.389889..), $\left(f_{1}, g_{1}\right)=(0.927682 . ., 0.372865 .),.\left(\gamma_{1}, \delta_{1}\right)=(3.15833 . .,-1.03889 .$.$) and$ $\left(h_{1}, k_{1}\right)=(0.434221 . .,-0.793941 .$.$) . These points satisfy equation e_{2}$ and $e_{3}$ of system (3.5) for $s=1$ and $i=2$.

The level curve corresponding to $k=2$ in $(I)$, intersects the cubic $c_{2}=0$ in $\left(\alpha_{2}, \beta_{2}\right)=$ (3.04069.., 0.502491..), $\left(f_{2}, g_{2}\right)=(0.878213 . ., 0.476377 .),.\left(\gamma_{2}, \delta_{2}\right)=(3.19002 . .,-1.15216 .$.$) and$ $\left(h_{2}, k_{2}\right)=(0.017634 . .,-0.227296 .$.$) . These points satisfy equation e_{2}$ and $e_{3}$ of system (3.5) for $s=2$ and $i=2$.

The level curve corresponding to $k=3$ in $(I)$, intersects $c_{2}=0$ in $\left(\alpha_{3}, \beta_{3}\right)=(3.05752 . ., 0.601532 .$.$) ,$ $\left(f_{3}, g_{3}\right)=(0.821437 . ., 0.565285 .),.\left(\gamma_{3}, \delta_{3}\right)=(3.21933 . .,-1.25183 .$.$) and \left(h_{2}, k_{2}\right)=(0.00229058 . ., 0.0827$ These points satisfy equation $e_{2}$ and $e_{3}$ of system (3.5) for $s=3$ and $i=2$.

In short, in the region $R_{1}$ we consider the linear differential center

$$
\dot{x}=-1.4-4 y, \dot{y}=-2+x,
$$

with its first integral $H_{2}(x, y)=4 x^{2}+8\left(\frac{7 y}{5}-2 x\right)+16 y^{2}$.

Now we have to give the expression of $H_{1}(x, y)$ in the region $R_{2}$ and $H_{3}(x, y)$ in the region $R_{3}$. Then we deal with the equations $e_{1}$ and $e_{4}$ in (3.5). By solving these equations for $s=1,2,3$, then fixing $b_{1}=-2 / 25$ in equation $e_{1}$ and $\sigma_{1}=-2$ in equation $e_{4}$, we obtain the Hamiltonian systems

$$
\begin{aligned}
\dot{x}= & -0.00039097 . . x-0.08 . . y-0.0252659 . ., \dot{y}=\frac{1.91072 . .}{10^{6}} x \\
& +0.00039097 . . y+0.00562837 . ., \text { in } R_{2}, \\
\dot{x}= & -0.0474089 . . x-0.000365066 . . y+0.955189 . ., \dot{y}=6.15669 . . x \\
& +0.0474089 . . y-2, \text { in } R_{3} .
\end{aligned}
$$


The Hamiltonian first integrals of the Hamiltonian systems (3.7) are

$$
\begin{aligned}
& H_{1}(x, y)=\frac{9.55359 x^{2}}{10^{7}}+0.00039097 . . x y+0.00562837 . . x+0.04 . . y^{2}+0.0252659 . . y \\
& H_{3}(x, y)=3.07835 . . x^{2}+0.0474089 . . x y-2 x+0.000182533 . . y^{2}-0.955189 . . y,
\end{aligned}
$$

For the discontinuous piecewise differential systems (3.6)-(3.7) all the real solutions of the system of equations (3.5) with $s=1,2,3$ and $i=2$ are

$$
\begin{aligned}
\left(\alpha_{1}, \beta_{1}, \gamma_{1}, \delta_{1}, f_{1}, g_{1}, h_{1}, k_{1}\right)= & (3.02482 . ., 0.389889 . ., 3.15833 . .,-1.03889 . . \\
& 0.927682 . ., 0.372865 . ., 0.434221 . .,-0.793941 . .), \\
\left(\alpha_{2}, \beta_{2}, \gamma_{2}, \delta_{2}, f_{2}, g_{2}, h_{2}, k_{2}\right)= & (3.04069 . ., 0.502491 . ., 3.19002 . .,-1.15216 . . \\
& 0.878213 . ., 0.476377 . ., 0.017634 . .,-0.227296 . .), \\
\left(\alpha_{3}, \beta_{3}, \gamma_{3}, \delta_{3}, f_{3}, g_{3}, h_{3}, k_{3}\right)= & (3.05752 . ., 0.601532 . ., 3.21933 . .,-1.25183 . . \\
& 0.821437 . ., 0.565285 . ., 0.00229058 . ., 0.0827693 . .),
\end{aligned}
$$

Then the discontinuous piecewise linear differential systems (3.6)-(3.7) have exactly three limit cycles, see $\left(C_{2}^{1}\right)$ of Figure 14.

Example 7. Three limit cycles intersecting the curve $c_{5}=0$ in four points. We consider the regions $R_{i}$ for $i=1,2,3$ defined in (2.26). In the region $R_{2}$ we consider the linear differential center

$$
\dot{x}=-\frac{x}{5}-\frac{629 y}{100}+\frac{2}{5}, \dot{y}=x+\frac{y}{5}-\frac{4}{5}
$$

its first integral is $H_{2}(x, y)=4\left(x+\frac{y}{5}\right)^{2}+8\left(-\frac{4 x}{5}-\frac{2 y}{5}\right)+25 y^{2}$.

Now we consider the following Hamiltonian systems

$$
\begin{aligned}
\dot{x}= & 29.8246 . . x+y-34.8532 . ., \dot{y}=-889.505 . . x-29.8246 . . y \\
& +97.1847 . ., \text { in } R_{1}, \\
\dot{x}= & 0.00341157 . . x+\frac{3 y}{2}-0.095202 . ., \dot{y}=-\frac{7.75919897096031}{10^{6}} x \\
& -0.00341157 . . y+0.198512 . ., \text { in } R_{3} .
\end{aligned}
$$

The Hamiltonian first integrals of the Hamiltonian systems (3.9) are

$$
\begin{aligned}
& H_{1}(x, y)=-444.753 . . x^{2}-29.8246 . . x y+97.1847 . . x-\frac{y^{2}}{2}+34.8532 . . y, \\
& H_{3}(x, y)=-\frac{3.8796}{10^{6}} x^{2}-0.00341157 . . x y+0.198512 . . x-\frac{3 y^{2}}{4}+0.095202 . . y,
\end{aligned}
$$

respectively. For the discontinuos piecewise linear differential systems (3.8)-(3.9) the real solutions of the system of equations (3.4) are

$$
\begin{aligned}
\left(\alpha_{1}, \beta_{1}, \gamma_{1}, \delta_{1}, f_{1}, g_{1}, h_{1}, k_{1}\right)= & (0.343158 . ., 0.397701 . ., 0.23703 . .,-0.263629 . . \\
& -0.163657 . ., 0.149667 . .,-0.0982745 . .,-0.0933207 . .), \\
\left(\alpha_{2}, \beta_{2}, \gamma_{2}, \delta_{2}, f_{2}, g_{2}, h_{2}, k_{2}\right)= & (0.396 . ., 0.467884 . ., 0.304088 . .,-0.347259 . . \\
& -0.261162 . ., 0.224484 . .,-0.173194 . .,-0.157483 . .), \\
\left(\alpha_{3}, \beta_{3}, \gamma_{3}, \delta_{3}, f_{3}, g_{3}, h_{3}, k_{3}\right)= & (0.438244 . ., 0.525572 . ., 0.354392 . .,-0.412436 . . \\
& -0.345135 . ., 0.279296 . .,-0.23907 . .,-0.208544 . .),
\end{aligned}
$$


where $\alpha_{i}=r_{i}^{2}-1, \beta_{i}=r_{i}\left(r_{i}^{2}-1\right), \gamma_{i}=s_{i}^{2}-1, \delta_{i}=s_{i}\left(s_{i}^{2}-1\right), a_{i}=f_{i}^{2}-1, b_{i}=f_{i}\left(f_{i}^{2}-1\right)$, $c_{i}=h_{i}^{2}-1, d_{i}=h_{i}\left(h_{i}^{2}-1\right)$.

Then the discontinuous piecewise linear differential systems (3.8)-(3.9) have exactly three limit cycles, see $\left(C_{5}^{1}\right)$ of Figure 16. This completes the proof of statement $(a)$ of Theorem 1.3.

Right now we start the proof of statement (b) of Theorem 1.3.

In order to have limit cycles with two and four intersection points with the cubic $c_{2}=0$ simultaneously, the points of intersection of the limit cycles in two points with $c_{2}=0$ must satisfy system (2.4), and the points of intersection of the limit cycles in four points with $c_{2}=0$ must satisfy system (3.4). In statement (ii) of Theorem 1.2 we proved that the maximum number of limit cycles with two intersection points with $c_{2}=0$ is three, and we also proved in the first statement of this Theorem that the maximum number of limit cycles with four intersection points with $c_{2}=0$ is three, then we have that the upper bound of maximum number of limit cycles with two and four intersection points, simultaneously, is six. This upper bound is reached.

Examples 8. Three limit cycles with four intersection points on $c_{2}=0$ and three limit cycles with two intersection points on $c_{2}=0$. Habitually we consider the regions defined in (2.11).

In the region $R_{1}$ we consider the linear differential center

$$
\dot{x}=0.0408657 . . x-0.18657 . . y-0.161702 . ., \dot{y}=x-0.0408657 . . y-2.22076 . .,
$$

its first integral is $H_{2}(x, y)=4(x-0.0413008 . . y)^{2}+8(0.163443 . . y-2.21892 . . x)+0.7569 . . y^{2}$.

Now we consider the Hamiltonian systems

$$
\begin{aligned}
\dot{x}= & 0.5 x-5 y-2, \dot{y}=0.05 x-0.5 y+2, \text { in } R_{2} \\
\dot{x}= & 0.275117 . . x+4 y-0.895428 . ., \dot{y}=-0.0189223 . . x \\
& -0.275117 . . y-10.3678 . ., \text { in } R_{3}
\end{aligned}
$$

The Hamiltonian first integrals of the Hamiltonian systems (3.11) are

$$
\begin{aligned}
& H_{1}(x, y)=0.025 x^{2}-0.5 x y+2 x+\frac{5 y^{2}}{2}+2 y \\
& H_{3}(x, y)=-0.00838913 . . x^{2}-0.28964 . . x y-12.0087 . . x-\frac{5 y^{2}}{2}+0.897776 . . y .
\end{aligned}
$$

The discontinuos piecewise differential systems (3.10)-(3.11) have three limit cycles intersecting the cubic $c_{2}=0$ in four points satisfying system (3.5) and three limit cycles intersecting the cubic $c_{2}=0$ in two points satisfying system (2.4), because all the real solutions of these two 
systems are

$$
\begin{aligned}
\left(\alpha_{1}, \beta_{1}, \gamma_{1}, \delta_{1}, f_{1}, g_{1}, h_{1}, k_{1}\right)= & (3.29854 . .,-1.50447 . ., 3.25648 . ., 1.37282 . ., \\
& 0.996543 . ., 0.0830817 . ., 0.967473 . .,-0.252974 . .), \\
\left(\alpha_{2}, \beta_{2}, \gamma_{2}, \delta_{2}, f_{2}, g_{2}, h_{2}, k_{2}\right)= & (3.33756 . .,-1.62283 . ., 3.29637 . ., 1.49782 . ., 0.959503 . ., \\
& 0.281699 . ., 0.897792 . .,-0.44042 . .), \\
\left(\alpha_{3}, \beta_{3}, \gamma_{3}, \delta_{3}, f_{3}, g_{3}, h_{3}, k_{3}\right)= & (3.37472 . .,-1.73293 . ., 3.33474 . ., 1.61438 . ., 0.916959 . ., \\
& 0.398981 . ., 0.836989 . .,-0.547219 . .), \\
\left(\alpha_{4}, \beta_{4}, \gamma_{4}, \delta_{4}\right)= & (3.1668 . .,-1.06984 . ., 3.12648 . ., 0.916988 . .), \\
\left(\alpha_{5}, \beta_{5}, \gamma_{5}, \delta_{5}\right)= & (3.21363 . .,-1.23276 . ., 3.17161 . ., 1.0872 . .), \\
\left(\alpha_{6}, \beta_{6}, \gamma_{6}, \delta_{6}\right)= & (3.25735 . .,-1.37562 . ., 3.21493 . ., 1.23712 . .) .
\end{aligned}
$$

Then the discontinuous piecewise differential systems (3.10)-(3.11) have exactly three limit cycles, see $\left(C_{2}^{2}\right)$ of Figure 15.

Example 9. Three limit cycles with four intersection points on $c_{5}=0$ and three limit cycles with two intersection points on $c_{5}=0$. In order to have limit cycles with two and four intersection points with the cubic $c_{5}=0$ simultaneously, the points of intersection of the limit cycles in two points with $c_{5}=0$ must satisfy system (2.4), and the points of intersection of the limit cycles in four points with $c_{5}=0$ must satisfy system (3.4). In statement (iii) of Theorem 1.2 we proved that the maximum number of limit cycles with two intersection points with $c_{5}=0$ is three, and we also proved in statement (i) of this Theorem that the maximum number of limit cycles with four intersection points with $c_{5}=0$ is three, then we have that the upper bound of the maximum number of limit cycles with two and four intersection points, simultaneously, is six. The sesult is reached in the following example.

Habitually we consider the regions defined in (2.26).

In the region $R_{2}$ we consider the linear differential center

$$
\begin{aligned}
\dot{x}= & 0.208169 . . x-0.0463593 . . y+0.0028092 . ., \dot{y}=x-0.208169 . . y \\
& -0.217992 . .,
\end{aligned}
$$

its first integral is $H_{2}(x, y)=4(x-0.208169 . . y)^{2}+8(-0.217992 . . x-0.0028092 . . y)+0.0121 y^{2}$.

Now we consider the Hamiltonian systems

$$
\begin{aligned}
\dot{x}= & 40 x-8 y+9, \dot{y}=-33+200 x-40 y, \text { in } R_{1}, \\
\dot{x}= & 54.173 . . x-6 y+31.215 . ., \dot{y}=489.119 . . x-54.173 . . y \\
& -0.0147783 . ., \text { in } R_{3},
\end{aligned}
$$

and their Hamiltonian first integrals

$$
\begin{aligned}
& H_{1}(x, y)=100 x^{2}-40 x y-33 x+4 y^{2}-9 y, \\
& H_{3}(x, y)=244.56 . . x^{2}-54.173 . . x y-0.0147783 . . x+3 y^{2}-31.215 . . y,
\end{aligned}
$$

respectively.

Due to the fact that system (3.4) has three real solutions and system (2.4) has three real solutions, it results that the discontinuos piecewise differential systems (3.12)-(3.13) have three limit cycles intersecting the cubic $c_{5}=0$ in four points and three limit cycles intersecting the 
cubic $c_{5}=0$ in two points. The real solutions of the two systems (3.4) and (2.4) are

$$
\begin{aligned}
\left(\alpha_{1}, \beta_{1}, \gamma_{1}, \delta_{1}, f_{1}, g_{1}, h_{1}, k_{1}\right)= & (0.294986 . .,-0.335687 . ., 1.01573 . ., 1.44209 . ., \\
& -0.113479 . ., 0.106846 . .,-0.0149546 . .,-0.0148423 . .), \\
\left(\alpha_{2}, \beta_{2}, \gamma_{2}, \delta_{2}, f_{2}, g_{2}, h_{2}, k_{2}\right)= & (0.34172 . .,-0.395823 . ., 1.09233 . ., 1.58004 . ., \\
& -0.159928 . ., 0.146583 . .,-0.0691478 . .,-0.0667143 . .), \\
\left(\alpha_{3}, \beta_{3}, \gamma_{3}, \delta_{3}, f_{3}, g_{3}, h_{3}, k_{3}\right)= & (0.34172 . .,-0.395823 . ., 1.09233 . ., 1.58004 . ., \\
& -0.200605 . ., 0.179359 . .,-0.119305 . .,-0.111962 . .), \\
\left(\alpha_{4}, \beta_{4}, \gamma_{4}, \delta_{4}\right)= & (0.203429 . ., 0.223163 . ., 0.620097 . ., 0.789278 . .), \\
\left(\alpha_{5}, \beta_{5}, \gamma_{5}, \delta_{5}\right)= & (0.126866 . ., 0.134673 . ., 0.705726 . ., 0.921702 . .), \\
\left(\alpha_{6}, \beta_{6}, \gamma_{6}, \delta_{6}\right)= & (0.0684267 . ., 0.070729 . ., 0.773252 . ., 1.02969 . .) .
\end{aligned}
$$

Then the discontinuous piecewise differential systems (3.12)-(3.13) have exactly six limit cycles, see $\left(C_{5}^{2}\right)$ of Figure 17.

This completes the proof of statement (b) of Theorem 1.3.

\section{The appendix} 1.2 .

Here we provide the values of $R_{1}, R_{2}, R_{3}, D_{1}$ and $D_{2}$ that appear in the proof of Theorem

$$
\begin{aligned}
& R_{1}=r_{1}^{4} r_{2}^{2} r_{3}-r_{1}^{2} r_{2}^{4} r_{3}-r_{1}^{4} r_{2} r_{3}^{2}+r_{1} r_{2}^{4} r_{3}^{2}+r_{1}^{2} r_{2} r_{3}^{4}-r_{1} r_{2}^{2} r_{3}^{4}+r_{1}^{3} r_{2}^{2} r_{3} s_{1}-r_{1} r_{2}^{4} r_{3} s_{1} \\
& -r_{1}^{3} r_{2} r_{3}^{2} s_{1}+r_{2}^{4} r_{3}^{2} s_{1}+r_{1} r_{2} r_{3}^{4} s_{1}-r_{2}^{2} r_{3}^{4} s_{1}+r_{1}^{2} r_{2}^{2} r_{3} s_{1}^{2}-r_{2}^{4} r_{3} s_{1}^{2}-r_{1}^{2} r_{2} r_{3}^{2} s_{1}^{2} \\
& +r_{2} r_{3}^{4} s_{1}^{2}+r_{1} r_{2}^{2} r_{3} s_{1}^{3}-r_{1} r_{2} r_{3}^{2} s_{1}^{3}+r_{2}^{2} r_{3} s_{1}^{4}-r_{2} r_{3}^{2} s_{1}^{4}+r_{1}^{4} r_{2} r_{3} s_{2}-r_{1}^{2} r_{2}^{3} r_{3} s_{2} \\
& -r_{1}^{4} r_{3}^{2} s_{2}+r_{1} r_{2}^{3} r_{3}^{2} s_{2}+r_{1}^{2} r_{3}^{4} s_{2}-r_{1} r_{2} r_{3}^{4} s_{2}+r_{1}^{3} r_{2} r_{3} s_{1} s_{2}-r_{1} r_{2}^{3} r_{3} s_{1} s_{2}-r_{1}^{3} r_{3}^{2} s_{1} s_{2} \\
& +r_{2}^{3} r_{3}^{2} s_{1} s_{2}+r_{1} r_{3}^{4} s_{1} s_{2}-r_{2} r_{3}^{4} s_{1} s_{2}+r_{1}^{2} r_{2} r_{3} s_{1}^{2} s_{2}-r_{2}^{3} r_{3} s_{1}^{2} s_{2}-r_{1}^{2} r_{3}^{2} s_{1}^{2} s_{2}+r_{3}^{4} s_{1}^{2} s_{2} \\
& +r_{1} r_{2} r_{3} s_{1}^{3} s_{2}-r_{1} r_{3}^{2} s_{1}^{3} s_{2}+r_{2} r_{3} s_{1}^{4} s_{2}-r_{3}^{2} s_{1}^{4} s_{2}+r_{1}^{4} r_{3} s_{2}^{2}-r_{1}^{2} r_{2}^{2} r_{3} s_{2}^{2}+r_{1} r_{2}^{2} r_{3}^{2} s_{2}^{2} \\
& -r_{1} r_{3}^{4} s_{2}^{2}+r_{1}^{3} r_{3} s_{1} s_{2}^{2}-r_{1} r_{2}^{2} r_{3} s_{1} s_{2}^{2}+r_{2}^{2} r_{3}^{2} s_{1} s_{2}^{2}-r_{3}^{4} s_{1} s_{2}^{2}+r_{1}^{2} r_{3} s_{1}^{2} s_{2}^{2}-r_{2}^{2} r_{3} s_{1}^{2} s_{2}^{2} \\
& +r_{1} r_{3} s_{1}^{3} s_{2}^{2}+r_{3} s_{1}^{4} s_{2}^{2}-r_{1}^{2} r_{2} r_{3} s_{2}^{3}+r_{1} r_{2} r_{3}^{2} s_{2}^{3}-r_{1} r_{2} r_{3} s_{1} s_{2}^{3}+r_{2} r_{3}^{2} s_{1} s_{2}^{3}-r_{2} r_{3} s_{1}^{2} s_{2}^{3} \\
& -r_{1}^{2} r_{3} s_{2}^{4}+r_{1} r_{3}^{2} s_{2}^{4}-r_{1} r_{3} s_{1} s_{2}^{4}+r_{3}^{2} s_{1} s_{2}^{4}-r_{3} s_{1}^{2} s_{2}^{4}+r_{1}^{4} r_{2}^{2} s_{3}-r_{1}^{2} r_{2}^{4} s_{3}-r_{1}^{4} r_{2} r_{3} s_{3} \\
& +r_{1} r_{2}^{4} r_{3} s_{3}+r_{1}^{2} r_{2} r_{3}^{3} s_{3}-r_{1} r_{2}^{2} r_{3}^{3} s_{3}+r_{1}^{3} r_{2}^{2} s_{1} s_{3}-r_{1} r_{2}^{4} s_{1} s_{3}-r_{1}^{3} r_{2} r_{3} s_{1} s_{3}+r_{2}^{4} r_{3} s_{1} s_{3} \\
& +r_{1} r_{2} r_{3}^{3} s_{1} s_{3}-r_{2}^{2} r_{3}^{3} s_{1} s_{3}+r_{1}^{2} r_{2}^{2} s_{1}^{2} s_{3}-r_{2}^{4} s_{1}^{2} s_{3}-r_{1}^{2} r_{2} r_{3} s_{1}^{2} s_{3}+r_{2} r_{3}^{3} s_{1}^{2} s_{3}+r_{1} r_{2}^{2} s_{1}^{3} s_{3} \\
& -r_{1} r_{2} r_{3} s_{1}^{3} s_{3}+r_{2}^{2} s_{1}^{4} s_{3}-r_{2} r_{3} s_{1}^{4} s_{3}+r_{1}^{4} r_{2} s_{2} s_{3}-r_{1}^{2} r_{2}^{3} s_{2} s_{3}-r_{1}^{4} r_{3} s_{2} s_{3}+r_{1} r_{2}^{3} r_{3} s_{2} s_{3} \\
& +r_{1}^{2} r_{3}^{3} s_{2} s_{3}-r_{1} r_{2} r_{3}^{3} s_{2} s_{3}+r_{1}^{3} r_{2} s_{1} s_{2} s_{3}-r_{1} r_{2}^{3} s_{1} s_{2} s_{3}-r_{1}^{3} r_{3} s_{1} s_{2} s_{3}+r_{2}^{3} r_{3} s_{1} s_{2} s_{3} \\
& +r_{1} r_{3}^{3} s_{1} s_{2} s_{3}-r_{2} r_{3}^{3} s_{1} s_{2} s_{3}+r_{1}^{2} r_{2} s_{1}^{2} s_{2} s_{3}-r_{2}^{3} s_{1}^{2} s_{2} s_{3}-r_{1}^{2} r_{3} s_{1}^{2} s_{2} s_{3}+r_{3}^{3} s_{1}^{2} s_{2} s_{3} \\
& +r_{1} r_{2} s_{1}^{3} s_{2} s_{3}-r_{1} r_{3} s_{1}^{3} s_{2} s_{3}+r_{2} s_{1}^{4} s_{2} s_{3}-r_{3} s_{1}^{4} s_{2} s_{3}+r_{1}^{4} s_{2}^{2} s_{3}-r_{1}^{2} r_{2}^{2} s_{2}^{2} s_{3}+r_{1} r_{2}^{2} r_{3} s_{2}^{2} s_{3} \\
& -r_{1} r_{3}^{3} s_{2}^{2} s_{3}+r_{1}^{3} s_{1} s_{2}^{2} s_{3}-r_{1} r_{2}^{2} s_{1} s_{2}^{2} s_{3}+r_{2}^{2} r_{3} s_{1} s_{2}^{2} s_{3}-r_{3}^{3} s_{1} s_{2}^{2} s_{3}+r_{1}^{2} s_{1}^{2} s_{2}^{2} s_{3}-r_{2}^{2} s_{1}^{2} s_{2}^{2} s_{3} \\
& +r_{1} s_{1}^{3} s_{2}^{2} s_{3}+s_{1}^{4} s_{2}^{2} s_{3}-r_{1}^{2} r_{2} s_{2}^{3} s_{3}+r_{1} r_{2} r_{3} s_{2}^{3} s_{3}-r_{1} r_{2} s_{1} s_{2}^{3} s_{3}+r_{2} r_{3} s_{1} s_{2}^{3} s_{3}-r_{2} s_{1}^{2} s_{2}^{3} s_{3} \\
& -r_{1}^{2} s_{2}^{4} s_{3}+r_{1} r_{3} s_{2}^{4} s_{3}-r_{1} s_{1} s_{2}^{4} s_{3}+r_{3} s_{1} s_{2}^{4} s_{3}-s_{1}^{2} s_{2}^{4} s_{3}-r_{1}^{4} r_{2} s_{3}^{2}+r_{1} r_{2}^{4} s_{3}^{2}+r_{1}^{2} r_{2} r_{3}^{2} s_{3}^{2} \\
& -r_{1} r_{2}^{2} r_{3}^{2} s_{3}^{2}-r_{1}^{3} r_{2} s_{1} s_{3}^{2}+r_{2}^{4} s_{1} s_{3}^{2}+r_{1} r_{2} r_{3}^{2} s_{1} s_{3}^{2}-r_{2}^{2} r_{3}^{2} s_{1} s_{3}^{2}-r_{1}^{2} r_{2} s_{1}^{2} s_{3}^{2}+r_{2} r_{3}^{2} s_{1}^{2} s_{3}^{2} \\
& -r_{1} r_{2} s_{1}^{3} s_{3}^{2}-r_{2} s_{1}^{4} s_{3}^{2}-r_{1}^{4} s_{2} s_{3}^{2}+r_{1} r_{2}^{3} s_{2} s_{3}^{2}+r_{1}^{2} r_{3}^{2} s_{2} s_{3}^{2}-r_{1} r_{2} r_{3}^{2} s_{2} s_{3}^{2}-r_{1}^{3} s_{1} s_{2} s_{3}^{2} \\
& +r_{2}^{3} s_{1} s_{2} s_{3}^{2}+r_{1} r_{3}^{2} s_{1} s_{2} s_{3}^{2}-r_{2} r_{3}^{2} s_{1} s_{2} s_{3}^{2}-r_{1}^{2} s_{1}^{2} s_{2} s_{3}^{2}+r_{3}^{2} s_{1}^{2} s_{2} s_{3}^{2}-r_{1} s_{1}^{3} s_{2} s_{3}^{2}-s_{1}^{4} s_{2} s_{3}^{2}
\end{aligned}
$$


$+r_{1} r_{2}^{2} s_{2}^{2} s_{3}^{2}-r_{1} r_{3}^{2} s_{2}^{2} s_{3}^{2}+r_{2}^{2} s_{1} s_{2}^{2} s_{3}^{2}-r_{3}^{2} s_{1} s_{2}^{2} s_{3}^{2}+r_{1} r_{2} s_{2}^{3} s_{3}^{2}+r_{2} s_{1} s_{2}^{3} s_{3}^{2}+r_{1} s_{2}^{4} s_{3}^{2}$ $+s_{1} s_{2}^{4} s_{3}^{2}+r_{1}^{2} r_{2} r_{3} s_{3}^{3}-r_{1} r_{2}^{2} r_{3} s_{3}^{3}+r_{1} r_{2} r_{3} s_{1} s_{3}^{3}-r_{2}^{2} r_{3} s_{1} s_{3}^{3}+r_{2} r_{3} s_{1}^{2} s_{3}^{3}+r_{1}^{2} r_{3} s_{2} s_{3}^{3}$ $-r_{1} r_{2} r_{3} s_{2} s_{3}^{3}+r_{1} r_{3} s_{1} s_{2} s_{3}^{3}-r_{2} r_{3} s_{1} s_{2} s_{3}^{3}+r_{3} s_{1}^{2} s_{2} s_{3}^{3}-r_{1} r_{3} s_{2}^{2} s_{3}^{3}-r_{3} s_{1} s_{2}^{2} s_{3}^{3}+r_{1}^{2} r_{2} s_{3}^{4}$ $-r_{1} r_{2}^{2} s_{3}^{4}+r_{1} r_{2} s_{1} s_{3}^{4}-r_{2}^{2} s_{1} s_{3}^{4}+r_{2} s_{1}^{2} s_{3}^{4}+r_{1}^{2} s_{2} s_{3}^{4}-r_{1} r_{2} s_{2} s_{3}^{4}+r_{1} s_{1} s_{2} s_{3}^{4}-r_{2} s_{1} s_{2} s_{3}^{4}$ $+s_{1}^{2} s_{2} s_{3}^{4}-r_{1} s_{2}^{2} s_{3}^{4}-s_{1} s_{2}^{2} s_{3}^{4}$

$R_{2}=4\left(r_{1}^{4}\left(r_{2}^{2}\left(r_{3}+s_{3}\right)+s_{2}\left(-r_{3}^{2}+r_{3}\left(s_{2}-s_{3}\right)+\left(s_{2}-s_{3}\right) s_{3}\right)-r_{2}\left(r_{3}^{2}+r_{3}\left(-s_{2}+s_{3}\right)+s_{3}\left(-s_{2}\right.\right.\right.\right.$ $\left.\left.\left.+s_{3}\right)\right)\right)+r_{1}^{3} s_{1}\left(r_{2}^{2}\left(r_{3}+s_{3}\right)+s_{2}\left(-r_{3}^{2}+r_{3}\left(s_{2}-s_{3}\right)+\left(s_{2}-s_{3}\right) s_{3}\right)-r_{2}\left(r_{3}^{2}+r_{3}\left(-s_{2}+s_{3}\right)\right.\right.$ $\left.\left.+s_{3}\left(-s_{2}+s_{3}\right)\right)\right)+r_{1}^{2}\left(-r_{2}^{4}\left(r_{3}+s_{3}\right)-r_{2}^{3} s_{2}\left(r_{3}+s_{3}\right)+r_{2}^{2}\left(s_{1}^{2}-s_{2}^{2}\right)\left(r_{3}+s_{3}\right)+r_{2}\left(r_{3}^{4}+r_{3}^{3} s_{3}\right.\right.$ $\left.+r_{3}^{2}\left(-s_{1}^{2}+s_{3}^{2}\right)+r_{3}\left(-s_{2}^{3}+s_{1}^{2}\left(s_{2}-s_{3}\right)+s_{3}^{3}\right)+s_{3}\left(-s_{2}^{3}+s_{1}^{2}\left(s_{2}-s_{3}\right)+s_{3}^{3}\right)\right)+s_{2}\left(r_{3}^{4}+r_{3}^{3} s_{3}\right.$ $\left.\left.+r_{3}^{2}\left(-s_{1}^{2}+s_{3}^{2}\right)+r_{3}\left(-s_{2}^{3}+s_{1}^{2}\left(s_{2}-s_{3}\right)+s_{3}^{3}\right)+s_{3}\left(-s_{2}^{3}+s_{1}^{2}\left(s_{2}-s_{3}\right)+s_{3}^{3}\right)\right)\right)+r_{1}\left(r_{2}^{4}\left(r_{3}^{2}\right.\right.$ $\left.+r_{3}\left(-s_{1}+s_{3}\right)+s_{3}\left(-s_{1}+s_{3}\right)\right)+r_{2}^{3} s_{2}\left(r_{3}^{2}+r_{3}\left(-s_{1}+s_{3}\right)+s_{3}\left(-s_{1}+s_{3}\right)\right)-r_{2}^{2}\left(r_{3}^{4}+r_{3}^{3} s_{3}\right.$ $\left.+r_{3}^{2}\left(-s_{2}^{2}+s_{3}^{2}\right)+r_{3}\left(-s_{1}^{3}+s_{1} s_{2}^{2}-s_{2}^{2} s_{3}+s_{3}^{3}\right)+s_{3}\left(-s_{1}^{3}+s_{1} s_{2}^{2}-s_{2}^{2} s_{3}+s_{3}^{3}\right)\right)+r_{2}\left(r_{3}^{4}\left(s_{1}\right.\right.$ $\left.-s_{2}\right)+r_{3}^{3}\left(s_{1}-s_{2}\right) s_{3}+r_{3}^{2}\left(-s_{1}^{3}+s_{2}^{3}+s_{1} s_{3}^{2}-s_{2} s_{3}^{2}\right)+r_{3}\left(s_{1}^{3}\left(s_{2}-s_{3}\right)+s_{2} s_{3}\left(s_{2}^{2}-s_{3}^{2}\right)+s_{1}\right.$ $\left.\left.\left(-s_{2}^{3}+s_{3}^{3}\right)\right)+s_{3}\left(s_{1}^{3}\left(s_{2}-s_{3}\right)+s_{2} s_{3}\left(s_{2}^{2}-s_{3}^{2}\right)+s_{1}\left(-s_{2}^{3}+s_{3}^{3}\right)\right)\right)+s_{2}\left(r_{3}^{4}\left(s_{1}-s_{2}\right)+r_{3}^{3}\left(s_{1}\right.\right.$ $\left.-s_{2}\right) s_{3}+r_{3}^{2}\left(-s_{1}^{3}+s_{2}^{3}+s_{1} s_{3}^{2}-s_{2} s_{3}^{2}\right)+r_{3}\left(s_{1}^{3}\left(s_{2}-s_{3}\right)+s_{2} s_{3}\left(s_{2}^{2}-s_{3}^{2}\right)+s_{1}\left(-s_{2}^{3}+s_{3}^{3}\right)\right)+s_{3}$ $\left.\left.\left(s_{1}^{3}\left(s_{2}-s_{3}\right)+s_{2} s_{3}\left(s_{2}^{2}-s_{3}^{2}\right)+s_{1}\left(-s_{2}^{3}+s_{3}^{3}\right)\right)\right)\right)+s_{1}\left(r_{2}^{4}\left(r_{3}^{2}+r_{3}\left(-s_{1}+s_{3}\right)+s_{3}\left(-s_{1}+s_{3}\right)\right)\right.$ $+r_{2}^{3} s_{2}\left(r_{3}^{2}+r_{3}\left(-s_{1}+s_{3}\right)+s_{3}\left(-s_{1}+s_{3}\right)\right)-r_{2}^{2}\left(r_{3}^{4}+r_{3}^{3} s_{3}+r_{3}^{2}\left(-s_{2}^{2}+s_{3}^{2}\right)+r_{3}\left(-s_{1}^{3}+s_{1} s_{2}^{2}\right.\right.$ $\left.\left.-s_{2}^{2} s_{3}+s_{3}^{3}\right)+s_{3}\left(-s_{1}^{3}+s_{1} s_{2}^{2}-s_{2}^{2} s_{3}+s_{3}^{3}\right)\right)+r_{2}\left(r_{3}^{4}\left(s_{1}-s_{2}\right)+r_{3}^{3}\left(s_{1}-s_{2}\right) s_{3}+r_{3}^{2}\left(-s_{1}^{3}+s_{2}^{3}\right.\right.$ $\left.+s_{1} s_{3}^{2}-s_{2} s_{3}^{2}\right)+r_{3}\left(s_{1}^{3}\left(s_{2}-s_{3}\right)+s_{2} s_{3}\left(s_{2}^{2}-s_{3}^{2}\right)+s_{1}\left(-s_{2}^{3}+s_{3}^{3}\right)\right)+s_{3}\left(s_{1}^{3}\left(s_{2}-s_{3}\right)+s_{2} s_{3}\left(s_{2}^{2}\right.\right.$ $\left.\left.\left.-s_{3}^{2}\right)+s_{1}\left(-s_{2}^{3}+s_{3}^{3}\right)\right)\right)+s_{2}\left(r_{3}^{4}\left(s_{1}-s_{2}\right)+r_{3}^{3}\left(s_{1}-s_{2}\right) s_{3}+r_{3}^{2}\left(-s_{1}^{3}+s_{2}^{3}+s_{1} s_{3}^{2}-s_{2} s_{3}^{2}\right)+r_{3}\left(s_{1}^{3}\right.\right.$ $\left.\left.\left.\left.\left(s_{2}-s_{3}\right)+s_{2} s_{3}\left(s_{2}^{2}-s_{3}^{2}\right)+s_{1}\left(-s_{2}^{3}+s_{3}^{3}\right)\right)+s_{3}\left(s_{1}^{3}\left(s_{2}-s_{3}\right)+s_{2} s_{3}\left(s_{2}^{2}-s_{3}^{2}\right)+s_{1}\left(-s_{2}^{3}+s_{3}^{3}\right)\right)\right)\right)\right)^{2}$ $-4\left(r_{1}^{2}\left(-r_{2}^{3}\left(r_{3}+s_{3}\right)+r_{2}^{2}\left(s_{1}-s_{2}\right)\left(r_{3}+s_{3}\right)+r_{2}\left(r_{3}^{3}+r_{3}\left(s_{1}-s_{2}-s_{3}\right)\left(s_{2}-s_{3}\right)+\left(s_{1}-s_{2}-s_{3}\right)\right.\right.\right.$ $\left.\left(s_{2}-s_{3}\right) s_{3}+r_{3}^{2}\left(-s_{1}+s_{3}\right)\right)+s_{2}\left(r_{3}^{3}+r_{3}\left(s_{1}-s_{2}-s_{3}\right)\left(s_{2}-s_{3}\right)+\left(s_{1}-s_{2}-s_{3}\right)\left(s_{2}-s_{3}\right) s_{3}\right.$ $\left.\left.+r_{3}^{2}\left(-s_{1}+s_{3}\right)\right)\right)+r_{1}^{3}\left(r_{2}^{2}\left(r_{3}+s_{3}\right)+s_{2}\left(-r_{3}^{2}+r_{3}\left(s_{2}-s_{3}\right)+\left(s_{2}-s_{3}\right) s_{3}\right)-r_{2}\left(r_{3}^{2}+r_{3}\left(-s_{2}+s_{3}\right)\right.\right.$ $\left.\left.+s_{3}\left(-s_{2}+s_{3}\right)\right)\right)+r_{1}\left(r_{2}\left(s_{1}-s_{2}\right)\left(r_{3}^{3}+r_{3}\left(s_{1}-s_{3}\right)\left(s_{2}-s_{3}\right)-r_{3}^{2}\left(s_{1}+s_{2}-s_{3}\right)+\left(s_{1}-s_{3}\right)\left(s_{2}\right.\right.\right.$ $\left.\left.-s_{3}\right) s_{3}\right)+\left(s_{1}-s_{2}\right) s_{2}\left(r_{3}^{3}+r_{3}\left(s_{1}-s_{3}\right)\left(s_{2}-s_{3}\right)-r_{3}^{2}\left(s_{1}+s_{2}-s_{3}\right)+\left(s_{1}-s_{3}\right)\left(s_{2}-s_{3}\right) s_{3}\right)$ $+r_{2}^{3}\left(r_{3}^{2}+r_{3}\left(-s_{1}+s_{3}\right)+s_{3}\left(-s_{1}+s_{3}\right)\right)-r_{2}^{2}\left(r_{3}^{3}+r_{3}^{2}\left(-s_{2}+s_{3}\right)-r_{3}\left(s_{1}-s_{3}\right)\left(s_{1}-s_{2}+s_{3}\right)\right.$ $\left.\left.-\left(s_{1}-s_{3}\right) s_{3}\left(s_{1}-s_{2}+s_{3}\right)\right)\right)+s_{1}\left(r_{2}\left(s_{1}-s_{2}\right)\left(r_{3}^{3}+r_{3}\left(s_{1}-s_{3}\right)\left(s_{2}-s_{3}\right)-r_{3}^{2}\left(s_{1}+s_{2}-s_{3}\right)\right.\right.$ $\left.+\left(s_{1}-s_{3}\right)\left(s_{2}-s_{3}\right) s_{3}\right)+\left(s_{1}-s_{2}\right) s_{2}\left(r_{3}^{3}+r_{3}\left(s_{1}-s_{3}\right)\left(s_{2}-s_{3}\right)-r_{3}^{2}\left(s_{1}+s_{2}-s_{3}\right)+\left(s_{1}-s_{3}\right)\right.$ $\left.\left(s_{2}-s_{3}\right) s_{3}\right)+r_{2}^{3}\left(r_{3}^{2}+r_{3}\left(-s_{1}+s_{3}\right)+s_{3}\left(-s_{1}+s_{3}\right)\right)-r_{2}^{2}\left(r_{3}^{3}+r_{3}^{2}\left(-s_{2}+s_{3}\right)-r_{3}\left(s_{1}-s_{3}\right)\left(s_{1}\right.\right.$ $\left.\left.\left.\left.-s_{2}+s_{3}\right)-\left(s_{1}-s_{3}\right) s_{3}\left(s_{1}-s_{2}+s_{3}\right)\right)\right)\right)\left(r_{1}^{5}\left(r_{2}^{2}\left(r_{3}+s_{3}\right)+s_{2}\left(-r_{3}^{2}+r_{3}\left(s_{2}-s_{3}\right)+\left(s_{2}-s_{3}\right) s_{3}\right)\right.\right.$ $\left.-r_{2}\left(r_{3}^{2}+r_{3}\left(-s_{2}+s_{3}\right)+s_{3}\left(-s_{2}+s_{3}\right)\right)\right)+r_{1}^{4} s_{1}\left(r_{2}^{2}\left(r_{3}+s_{3}\right)+s_{2}\left(-r_{3}^{2}+r_{3}\left(s_{2}-s_{3}\right)+\left(s_{2}-s_{3}\right)\right.\right.$ $\left.\left.s_{3}\right)-r_{2}\left(r_{3}^{2}+r_{3}\left(-s_{2}+s_{3}\right)+s_{3}\left(-s_{2}+s_{3}\right)\right)\right)+r_{1}^{3} s_{1}^{2}\left(r_{2}^{2}\left(r_{3}+s_{3}\right)+s_{2}\left(-r_{3}^{2}+r_{3}\left(s_{2}-s_{3}\right)+\left(s_{2}\right.\right.\right.$ $\left.\left.\left.-s_{3}\right) s_{3}\right)-r_{2}\left(r_{3}^{2}+r_{3}\left(-s_{2}+s_{3}\right)+s_{3}\left(-s_{2}+s_{3}\right)\right)\right)+r_{1}^{2}\left(-r_{2}^{5}\left(r_{3}+s_{3}\right)-r_{2}^{4} s_{2}\left(r_{3}+s_{3}\right)-r_{2}^{3} s_{2}^{2}\left(r_{3}\right.\right.$ $\left.+s_{3}\right)+r_{2}^{2}\left(s_{1}^{3}-s_{2}^{3}\right)\left(r_{3}+s_{3}\right)+r_{2}\left(r_{3}^{5}+r_{3}^{4} s_{3}+r_{3}^{3} s_{3}^{2}+r_{3}^{2}\left(-s_{1}^{3}+s_{3}^{3}\right)+r_{3}\left(-s_{2}^{4}+s_{1}^{3}\left(s_{2}-s_{3}\right)+s_{3}^{4}\right)\right.$ $\left.+s_{3}\left(-s_{2}^{4}+s_{1}^{3}\left(s_{2}-s_{3}\right)+s_{3}^{4}\right)\right)+s_{2}\left(r_{3}^{5}+r_{3}^{4} s_{3}+r_{3}^{3} s_{3}^{2}+r_{3}^{2}\left(-s_{1}^{3}+s_{3}^{3}\right)+r_{3}\left(-s_{2}^{4}+s_{1}^{3}\left(s_{2}-s_{3}\right)\right.\right.$ $\left.\left.\left.+s_{3}^{4}\right)+s_{3}\left(-s_{2}^{4}+s_{1}^{3}\left(s_{2}-s_{3}\right)+s_{3}^{4}\right)\right)\right)+r_{1}\left(r_{2}^{5}\left(r_{3}^{2}+r_{3}\left(-s_{1}+s_{3}\right)+s_{3}\left(-s_{1}+s_{3}\right)\right)+r_{2}^{4} s_{2}\left(r_{3}^{2}\right.\right.$ $\left.+r_{3}\left(-s_{1}+s_{3}\right)+s_{3}\left(-s_{1}+s_{3}\right)\right)+r_{2}^{3} s_{2}^{2}\left(r_{3}^{2}+r_{3}\left(-s_{1}+s_{3}\right)+s_{3}\left(-s_{1}+s_{3}\right)\right)-r_{2}^{2}\left(r_{3}^{5}+r_{3}^{4} s_{3}+r_{3}^{3}\right.$ $\left.s_{3}^{2}+r_{3}^{2}\left(-s_{2}^{3}+s_{3}^{3}\right)+r_{3}\left(-s_{1}^{4}+s_{1} s_{2}^{3}-s_{2}^{3} s_{3}+s_{3}^{4}\right)+s_{3}\left(-s_{1}^{4}+s_{1} s_{2}^{3}-s_{2}^{3} s_{3}+s_{3}^{4}\right)\right)+r_{2}\left(r_{3}^{5}\left(s_{1}-s_{2}\right)\right.$ $+r_{3}^{4}\left(s_{1}-s_{2}\right) s_{3}+r_{3}^{3}\left(s_{1}-s_{2}\right) s_{3}^{2}+r_{3}^{2}\left(-s_{1}^{4}+s_{2}^{4}+s_{1} s_{3}^{3}-s_{2} s_{3}^{3}\right)+r_{3}\left(s_{1}^{4}\left(s_{2}-s_{3}\right)+s_{2} s_{3}\left(s_{2}^{3}-s_{3}^{3}\right)\right.$ $\left.\left.+s_{1}\left(-s_{2}^{4}+s_{3}^{4}\right)\right)+s_{3}\left(s_{1}^{4}\left(s_{2}-s_{3}\right)+s_{2} s_{3}\left(s_{2}^{3}-s_{3}^{3}\right)+s_{1}\left(-s_{2}^{4}+s_{3}^{4}\right)\right)\right)+s_{2}\left(r_{3}^{5}\left(s_{1}-s_{2}\right)+r_{3}^{4}\left(s_{1}\right.\right.$ $\left.-s_{2}\right) s_{3}+r_{3}^{3}\left(s_{1}-s_{2}\right) s_{3}^{2}+r_{3}^{2}\left(-s_{1}^{4}+s_{2}^{4}+s_{1} s_{3}^{3}-s_{2} s_{3}^{3}\right)+r_{3}\left(s_{1}^{4}\left(s_{2}-s_{3}\right)+s_{2} s_{3}\left(s_{2}^{3}-s_{3}^{3}\right)+s_{1}\left(-s_{2}^{4}\right.\right.$ $\left.\left.\left.\left.+s_{3}^{4}\right)\right)+s_{3}\left(s_{1}^{4}\left(s_{2}-s_{3}\right)+s_{2} s_{3}\left(s_{2}^{3}-s_{3}^{3}\right)+s_{1}\left(-s_{2}^{4}+s_{3}^{4}\right)\right)\right)\right)+s_{1}\left(r_{2}^{5}\left(r_{3}^{2}+r_{3}\left(-s_{1}+s_{3}\right)+s_{3}\left(-s_{1}\right.\right.\right.$ $\left.\left.+s_{3}\right)\right)+r_{2}^{4} s_{2}\left(r_{3}^{2}+r_{3}\left(-s_{1}+s_{3}\right)+s_{3}\left(-s_{1}+s_{3}\right)\right)+r_{2}^{3} s_{2}^{2}\left(r_{3}^{2}+r_{3}\left(-s_{1}+s_{3}\right)+s_{3}\left(-s_{1}+s_{3}\right)\right)$ 
$-r_{2}^{2}\left(r_{3}^{5}+r_{3}^{4} s_{3}+r_{3}^{3} s_{3}^{2}+r_{3}^{2}\left(-s_{2}^{3}+s_{3}^{3}\right)+r_{3}\left(-s_{1}^{4}+s_{1} s_{2}^{3}-s_{2}^{3} s_{3}+s_{3}^{4}\right)+s_{3}\left(-s_{1}^{4}+s_{1} s_{2}^{3}-s_{2}^{3} s_{3}\right.\right.$ $\left.\left.+s_{3}^{4}\right)\right)+r_{2}\left(r_{3}^{5}\left(s_{1}-s_{2}\right)+r_{3}^{4}\left(s_{1}-s_{2}\right) s_{3}+r_{3}^{3}\left(s_{1}-s_{2}\right) s_{3}^{2}+r_{3}^{2}\left(-s_{1}^{4}+s_{2}^{4}+s_{1} s_{3}^{3}-s_{2} s_{3}^{3}\right)+r_{3}\left(s_{1}^{4}\left(s_{2}\right.\right.\right.$ $\left.\left.\left.-s_{3}\right)+s_{2} s_{3}\left(s_{2}^{3}-s_{3}^{3}\right)+s_{1}\left(-s_{2}^{4}+s_{3}^{4}\right)\right)+s_{3}\left(s_{1}^{4}\left(s_{2}-s_{3}\right)+s_{2} s_{3}\left(s_{2}^{3}-s_{3}^{3}\right)+s_{1}\left(-s_{2}^{4}+s_{3}^{4}\right)\right)\right)+s_{2}$ $\left(r_{3}^{5}\left(s_{1}-s_{2}\right)+r_{3}^{4}\left(s_{1}-s_{2}\right) s_{3}+r_{3}^{3}\left(s_{1}-s_{2}\right) s_{3}^{2}+r_{3}^{2}\left(-s_{1}^{4}+s_{2}^{4}+s_{1} s_{3}^{3}-s_{2} s_{3}^{3}\right)+r_{3}\left(s_{1}^{4}\left(s_{2}-s_{3}\right)\right.\right.$ $\left.\left.\left.\left.+s_{2} s_{3}\left(s_{2}^{3}-s_{3}^{3}\right)+s_{1}\left(-s_{2}^{4}+s_{3}^{4}\right)\right)+s_{3}\left(s_{1}^{4}\left(s_{2}-s_{3}\right)+s_{2} s_{3}\left(s_{2}^{3}-s_{3}^{3}\right)+s_{1}\left(-s_{2}^{4}+s_{3}^{4}\right)\right)\right)\right)\right)$,

$$
\begin{aligned}
R_{3}= & r_{1}^{2}\left(-r_{2}^{3}\left(r_{3}+s_{3}\right)+r_{2}^{2}\left(s_{1}-s_{2}\right)\left(r_{3}+s_{3}\right)+r_{2}\left(r_{3}^{3}+r_{3}\left(s_{1}-s_{2}-s_{3}\right)\left(s_{2}-s_{3}\right)+\left(s_{1}-s_{2}-s_{3}\right)\right.\right. \\
& \left.\left(s_{2}-s_{3}\right) s_{3}+r_{3}^{2}\left(-s_{1}+s_{3}\right)\right)+s_{2}\left(r_{3}^{3}+r_{3}\left(s_{1}-s_{2}-s_{3}\right)\left(s_{2}-s_{3}\right)+\left(s_{1}-s_{2}-s_{3}\right)\left(s_{2}-s_{3}\right) s_{3}\right. \\
& \left.\left.+r_{3}^{2}\left(-s_{1}+s_{3}\right)\right)\right)+r_{1}^{3}\left(r_{2}^{2}\left(r_{3}+s_{3}\right)+s_{2}\left(-r_{3}^{2}+r_{3}\left(s_{2}-s_{3}\right)+\left(s_{2}-s_{3}\right) s_{3}\right)-r_{2}\left(r_{3}^{2}+r_{3}\left(-s_{2}\right.\right.\right. \\
& \left.\left.\left.+s_{3}\right)+s_{3}\left(-s_{2}+s_{3}\right)\right)\right)+r_{1}\left(r _ { 2 } ( s _ { 1 } - s _ { 2 } ) \left(r_{3}^{3}+r_{3}\left(s_{1}-s_{3}\right)\left(s_{2}-s_{3}\right)-r_{3}^{2}\left(s_{1}+s_{2}-s_{3}\right)\right.\right. \\
& \left.+\left(s_{1}-s_{3}\right)\left(s_{2}-s_{3}\right) s_{3}\right)+\left(s_{1}-s_{2}\right) s_{2}\left(r_{3}^{3}+r_{3}\left(s_{1}-s_{3}\right)\left(s_{2}-s_{3}\right)-r_{3}^{2}\left(s_{1}+s_{2}-s_{3}\right)+\left(s_{1}-s_{3}\right)\right. \\
& \left.\left(s_{2}-s_{3}\right) s_{3}\right)+r_{2}^{3}\left(r_{3}^{2}+r_{3}\left(-s_{1}+s_{3}\right)+s_{3}\left(-s_{1}+s_{3}\right)\right)-r_{2}^{2}\left(r_{3}^{3}+r_{3}^{2}\left(-s_{2}+s_{3}\right)-r_{3}\left(s_{1}-s_{3}\right)\left(s_{1}\right.\right. \\
& \left.\left.\left.-s_{2}+s_{3}\right)-\left(s_{1}-s_{3}\right) s_{3}\left(s_{1}-s_{2}+s_{3}\right)\right)\right)+s_{1}\left(r _ { 2 } ( s _ { 1 } - s _ { 2 } ) \left(r_{3}^{3}+r_{3}\left(s_{1}-s_{3}\right)\left(s_{2}-s_{3}\right)-r_{3}^{2}\left(s_{1}\right.\right.\right. \\
& \left.\left.+s_{2}-s_{3}\right)+\left(s_{1}-s_{3}\right)\left(s_{2}-s_{3}\right) s_{3}\right)+\left(s_{1}-s_{2}\right) s_{2}\left(r_{3}^{3}+r_{3}\left(s_{1}-s_{3}\right)\left(s_{2}-s_{3}\right)-r_{3}^{2}\left(s_{1}+s_{2}-s_{3}\right)\right. \\
& \left.+\left(s_{1}-s_{3}\right)\left(s_{2}-s_{3}\right) s_{3}\right)+r_{2}^{3}\left(r_{3}^{2}+r_{3}\left(-s_{1}+s_{3}\right)+s_{3}\left(-s_{1}+s_{3}\right)\right)-r_{2}^{2}\left(r_{3}^{3}+r_{3}^{2}\left(-s_{2}+s_{3}\right)-r_{3}\right. \\
& \left.\left.\left(s_{1}-s_{3}\right)\left(s_{1}-s_{2}+s_{3}\right)-\left(s_{1}-s_{3}\right) s_{3}\left(s_{1}-s_{2}+s_{3}\right)\right)\right) .
\end{aligned}
$$

$$
\begin{aligned}
D_{1}= & \frac{1}{-\left(r_{1}+s_{1}\right) s_{2}^{2}+\left(r_{1}^{2}+\left(s_{1}-r_{2}\right) r_{1}+s_{1}\left(s_{1}-r_{2}\right)\right) s_{2}+r_{2}\left(r_{1}^{2}+\left(s_{1}-r_{2}\right) r_{1}+s_{1}\left(s_{1}-r_{2}\right)\right)} \\
& \left(\left(b_{2}^{2}\left(-\left(r_{2}+s_{2}\right) s_{3}^{3}+\left(r_{2}^{2}+s_{2} r_{2}+s_{2}^{2}\right) s_{3}^{2}-r_{3}^{2}\left(r_{2}^{2}+\left(s_{2}-r_{3}\right) r_{2}+s_{2}\left(s_{2}-r_{3}\right)\right)\right) r_{1}^{5}+b_{2}\right.\right.
\end{aligned}
$$
$\left(b_{2} s_{1}+2\right)\left(r_{3}-s_{3}\right)\left(\left(r_{3}^{2}+\left(s_{3}-s_{2}\right) r_{3}+s_{3}\left(s_{3}-s_{2}\right)\right) r_{2}-r_{2}^{2}\left(r_{3}+s_{3}\right)+s_{2}\left(r_{3}^{2}+\left(s_{3}-s_{2}\right) r_{3}\right.\right.$ $\left.\left.+s_{3}\left(s_{3}-s_{2}\right)\right)\right) r_{1}^{4}+\left(b_{2} s_{1}+1\right)^{2}\left(r_{3}-s_{3}\right)\left(\left(r_{3}^{2}+\left(s_{3}-s_{2}\right) r_{3}+s_{3}\left(s_{3}-s_{2}\right)\right) r_{2}-r_{2}^{2}\left(r_{3}+s_{3}\right)\right.$ $\left.+s_{2}\left(r_{3}^{2}+\left(s_{3}-s_{2}\right) r_{3}+s_{3}\left(s_{3}-s_{2}\right)\right)\right) r_{1}^{3}+\left(r_{3}-s_{3}\right)\left(b_{2}^{2}\left(r_{3}+s_{3}\right) r_{2}^{5}+b_{2}\left(b_{2} s_{2}+2\right)\left(r_{3}+s_{3}\right) r_{2}^{4}\right.$ $+\left(b_{2} s_{2}+1\right)^{2}\left(r_{3}+s_{3}\right) r_{2}^{3}-\left(s_{1}-s_{2}\right)\left(\left(s_{1}^{2}+s_{2} s_{1}+s_{2}^{2}\right) b_{2}^{2}+2\left(s_{1}+s_{2}\right) b_{2}+1\right)\left(r_{3}+s_{3}\right) r_{2}^{2}$ $+\left(-2 b_{2} s_{3}^{4}-2 b_{2} r_{3} s_{3}^{3}+\left(-2 b_{2} r_{3}^{2}+s_{1}+b_{2} s_{1}^{2}\left(b_{2} s_{1}+2\right)\right) s_{3}^{2}+\left(b_{2}^{2} s_{2}^{4}+2 b_{2} s_{2}^{3}+s_{2}^{2}-s_{1}\left(b_{2} s_{1}\right.\right.\right.$ $\left.+1)^{2} s_{2}+r_{3}\left(-2 b_{2} r_{3}^{2}+s_{1}+b_{2} s_{1}^{2}\left(b_{2} s_{1}+2\right)\right)\right) s_{3}+r_{3}\left(b_{2}^{2} s_{2}^{4}+2 b_{2} s_{2}^{3}+s_{2}^{2}-s_{1}\left(b_{2} s_{1}+1\right)^{2} s_{2}\right.$ $\left.\left.+r_{3}\left(-2 b_{2} r_{3}^{2}+s_{1}+b_{2} s_{1}^{2}\left(b_{2} s_{1}+2\right)\right)\right)\right) r_{2}+s_{2}\left(-2 b_{2} s_{3}^{4}-2 b_{2} r_{3} s_{3}^{3}+\left(-2 b_{2} r_{3}^{2}+s_{1}+b_{2} s_{1}^{2}\left(b_{2} s_{1}\right.\right.\right.$ $+2)) s_{3}^{2}+\left(b_{2}^{2} s_{2}^{4}+2 b_{2} s_{2}^{3}+s_{2}^{2}-s_{1}\left(b_{2} s_{1}+1\right)^{2} s_{2}+r_{3}\left(-2 b_{2} r_{3}^{2}+s_{1}+b_{2} s_{1}^{2}\left(b_{2} s_{1}+2\right)\right)\right) s_{3}+r_{3}$ $\left.\left.\left(b_{2}^{2} s_{2}^{4}+2 b_{2} s_{2}^{3}+s_{2}^{2}-s_{1}\left(b_{2} s_{1}+1\right)^{2} s_{2}+r_{3}\left(-2 b_{2} r_{3}^{2}+s_{1}+b_{2} s_{1}^{2}\left(b_{2} s_{1}+2\right)\right)\right)\right)\right) r_{1}^{2}+\left(b_{2}^{2}\left(-r_{3}^{3}\right.\right.$ $\left.+s_{1} r_{3}^{2}+s_{3}^{2}\left(s_{3}-s_{1}\right)\right) r_{2}^{5}-b_{2}\left(b_{2} s_{2}+2\right)\left(r_{3}-s_{3}\right)\left(r_{3}^{2}+\left(s_{3}-s_{1}\right) r_{3}+s_{3}\left(s_{3}-s_{1}\right)\right) r_{2}^{4}-\left(b_{2} s_{2}+1\right)^{2}$ $\left(r_{3}-s_{3}\right)\left(r_{3}^{2}+\left(s_{3}-s_{1}\right) r_{3}+s_{3}\left(s_{3}-s_{1}\right)\right) r_{2}^{3}+\left(-2 b_{2} s_{3}^{5}+s_{2}\left(b_{2} s_{2}+1\right)^{2} s_{3}^{3}+s_{1}\left(b_{2}^{2} s_{1}^{3}+2 b_{2} s_{1}^{2}+s_{1}\right.\right.$ $\left.-s_{2}\left(b_{2} s_{2}+1\right)^{2}\right) s_{3}^{2}-r_{3}^{2}\left(-2 b_{2} r_{3}^{3}+b_{2}^{2}\left(r_{3}-s_{1}\right) s_{2}^{3}+s_{1}^{2}+2 b_{2}\left(r_{3}-s_{1}\right) s_{2}^{2}+b_{2} s_{1}^{3}\left(b_{2} s_{1}+2\right)+\left(r_{3}\right.\right.$ $\left.\left.\left.-s_{1}\right) s_{2}\right)\right) r_{2}^{2}+\left(s_{1}-s_{2}\right)\left(2 b_{2} s_{3}^{5}-\left(b_{2} s_{1}^{2}+s_{1}+b_{2} s_{2}^{2}+s_{2}\right)\left(b_{2}\left(s_{1}+s_{2}\right)+1\right) s_{3}^{3}+s_{1} s_{2}\left(\left(s_{1}^{2}+s_{2} s_{1}\right.\right.\right.$ $\left.\left.+s_{2}^{2}\right) b_{2}^{2}+2\left(s_{1}+s_{2}\right) b_{2}+1\right) s_{3}^{2}+r_{3}^{2}\left(\left(r_{3}\left(s_{1}+s_{2}\right)\left(s_{1}^{2}+s_{2}^{2}\right)-s_{1} s_{2}\left(s_{1}^{2}+s_{2} s_{1}+s_{2}^{2}\right)\right) b_{2}^{2}-2\left(r_{3}-s_{1}\right)\right.$ $\left.\left.\left(r_{3}-s_{2}\right)\left(r_{3}+s_{1}+s_{2}\right) b_{2}-s_{1} s_{2}+r_{3}\left(s_{1}+s_{2}\right)\right)\right) r_{2}+s_{2}\left(s_{2}-s_{1}\right)\left(-2 b_{2} s_{3}^{5}+\left(b_{2} s_{1}^{2}+s_{1}+b_{2} s_{2}^{2}\right.\right.$ $\left.+s_{2}\right)\left(b_{2}\left(s_{1}+s_{2}\right)+1\right) s_{3}^{3}-s_{1} s_{2}\left(\left(s_{1}^{2}+s_{2} s_{1}+s_{2}^{2}\right) b_{2}^{2}+2\left(s_{1}+s_{2}\right) b_{2}+1\right) s_{3}^{2}+r_{3}^{2}\left(-r_{3}\left(s_{1}+s_{2}\right)\right.$ $\left(s_{1}^{2}+s_{2}^{2}\right) b_{2}^{2}+s_{1} s_{2}\left(s_{1}^{2}+s_{2} s_{1}+s_{2}^{2}\right) b_{2}^{2}+2\left(r_{3}-s_{1}\right)\left(r_{3}-s_{2}\right)\left(r_{3}+s_{1}+s_{2}\right) b_{2}+s_{1} s_{2}-r_{3}\left(s_{1}\right.$ $\left.\left.\left.+s_{2}\right)\right)\right) r_{1}+s_{1}\left(b_{2}^{2}\left(-r_{3}^{3}+s_{1} r_{3}^{2}+s_{3}^{2}\left(s_{3}-s_{1}\right)\right) r_{2}^{5}-b_{2}\left(b_{2} s_{2}+2\right)\left(r_{3}-s_{3}\right)\left(r_{3}^{2}+\left(s_{3}-s_{1}\right) r_{3}+s_{3}\left(s_{3}\right.\right.\right.$ $\left.\left.-s_{1}\right)\right) r_{2}^{4}-\left(b_{2} s_{2}+1\right)^{2}\left(r_{3}-s_{3}\right)\left(r_{3}^{2}+\left(s_{3}-s_{1}\right) r_{3}+s_{3}\left(s_{3}-s_{1}\right)\right) r_{2}^{3}+\left(-2 b_{2} s_{3}^{5}+s_{2}\left(b_{2} s_{2}+1\right)^{2} s_{3}^{3}\right.$ 


$$
\begin{aligned}
& +s_{1}\left(b_{2}^{2} s_{1}^{3}+2 b_{2} s_{1}^{2}+s_{1}-s_{2}\left(b_{2} s_{2}+1\right)^{2}\right) s_{3}^{2}-r_{3}^{2}\left(-2 b_{2} r_{3}^{3}+b_{2}^{2}\left(r_{3}-s_{1}\right) s_{2}^{3}+s_{1}^{2}+2 b_{2}\left(r_{3}-s_{1}\right) s_{2}^{2}\right. \\
& \left.\left.+b_{2} s_{1}^{3}\left(b_{2} s_{1}+2\right)+\left(r_{3}-s_{1}\right) s_{2}\right)\right) r_{2}^{2}+\left(s_{1}-s_{2}\right)\left(2 b_{2} s_{3}^{5}-\left(b_{2} s_{1}^{2}+s_{1}+b_{2} s_{2}^{2}+s_{2}\right)\left(b_{2}\left(s_{1}+s_{2}\right)+1\right) s_{3}^{3}\right. \\
& +s_{1} s_{2}\left(\left(s_{1}^{2}+s_{2} s_{1}+s_{2}^{2}\right) b_{2}^{2}+2\left(s_{1}+s_{2}\right) b_{2}+1\right) s_{3}^{2}+r_{3}^{2}\left(\left(r_{3}\left(s_{1}+s_{2}\right)\left(s_{1}^{2}+s_{2}^{2}\right)-s_{1} s_{2}\left(s_{1}^{2}+s_{2} s_{1}+s_{2}^{2}\right)\right)\right. \\
& \left.\left.b_{2}^{2}-2\left(r_{3}-s_{1}\right)\left(r_{3}-s_{2}\right)\left(r_{3}+s_{1}+s_{2}\right) b_{2}-s_{1} s_{2}+r_{3}\left(s_{1}+s_{2}\right)\right)\right) r_{2}+s_{2}\left(s_{2}-s_{1}\right)\left(-2 b_{2} s_{3}^{5}+\left(b_{2} s_{1}^{2}\right.\right. \\
& \left.+s_{1}+b_{2} s_{2}^{2}+s_{2}\right)\left(b_{2}\left(s_{1}+s_{2}\right)+1\right) s_{3}^{3}-s_{1} s_{2}\left(\left(s_{1}^{2}+s_{2} s_{1}+s_{2}^{2}\right) b_{2}^{2}+2\left(s_{1}+s_{2}\right) b_{2}+1\right) s_{3}^{2}+r_{3}^{2}\left(-r_{3}\left(s_{1}\right.\right. \\
& \left.+s_{2}\right)\left(s_{1}^{2}+s_{2}^{2}\right) b_{2}^{2}+s_{1} s_{2}\left(s_{1}^{2}+s_{2} s_{1}+s_{2}^{2}\right) b_{2}^{2}+2\left(r_{3}-s_{1}\right)\left(r_{3}-s_{2}\right)\left(r_{3}+s_{1}+s_{2}\right) b_{2}+s_{1} s_{2} \\
& \left.\left.\left.\left.\left.-r_{3}\left(s_{1}+s_{2}\right)\right)\right)\right)\right)\right)+\left(s_{3}^{4}-r_{3}^{4}\right) a^{2}+b_{2}^{2}\left(s_{3}^{6}-r_{3}^{6}\right) \text {. } \\
& D_{2}=\frac{1}{s_{2}\left(r_{1}^{2}+r_{1}\left(s_{1}-r_{2}\right)+s_{1}\left(s_{1}-r_{2}\right)\right)+r_{2}\left(r_{1}^{2}+r_{1}\left(s_{1}-r_{2}\right)+s_{1}\left(s_{1}-r_{2}\right)\right)+s_{2}^{2}\left(-\left(r_{1}+s_{1}\right)\right)} \\
& \left(-r_{3}^{3}\left(r_{1}+s_{1}\right)\left(r_{2}+s_{2}\right)\left(r_{1}^{4}+r_{1}^{2} s_{1}^{2}-r_{2}^{4}-r_{2}^{2} s_{2}^{2}+s_{1}^{4}-s_{2}^{4}\right)+a r_{3}^{2}\left(r_{1}^{2}+r_{1} s_{1}+s_{1}^{2}\right)\left(r_{2}^{2}+r_{2} s_{2}\right.\right. \\
& \left.+s_{2}^{2}\right)\left(r_{1}^{3}-r_{2}^{3}+s_{1}^{3}-s_{2}^{3}\right)+a s_{3}^{2}\left(s_{3}\left(r_{1}+s_{1}\right)\left(r_{2}+s_{2}\right)\left(r_{1}^{4}+r_{1}^{2} s_{1}^{2}-r_{2}^{4}-r_{2}^{2} s_{2}^{2}+s_{1}^{4}-s_{2}^{4}\right)+\left(r_{1}^{2}\right.\right. \\
& \left.\left.+r_{1} s_{1}+s_{1}^{2}\right)\left(r_{2}^{2}+r_{2} s_{2}+s_{2}^{2}\right)\left(-r_{1}^{3}+r_{2}^{3}-s_{1}^{3}+s_{2}^{3}\right)\right)+s_{3}^{6}\left(-r_{1}^{2}\left(r_{2}+s_{2}\right)+r_{1}\left(r_{2}^{2}+r_{2}\left(s_{2}-s_{1}\right)\right.\right. \\
& \left.\left.+s_{2}\left(s_{2}-s_{1}\right)\right)+s_{1}\left(r_{2}^{2}+r_{2}\left(s_{2}-s_{1}\right)+s_{2}\left(s_{2}-s_{1}\right)\right)\right)+r_{3}^{6}\left(s_{2}\left(r_{1}^{2}+r_{1}\left(s_{1}-r_{2}\right)+s_{1}\left(s_{1}-r_{2}\right)\right)\right. \\
& \left.+r_{2}\left(r_{1}^{2}+r_{1}\left(s_{1}-r_{2}\right)+s_{1}\left(s_{1}-r_{2}\right)\right)+s_{2}^{2}\left(-\left(r_{1}+s_{1}\right)\right)\right) \text {. }
\end{aligned}
$$

\section{References}

[1] A. Andronov, A. Vitt and S. Khaikin, Theory of Oscillations, Pergamon Press, Oxford, 1966.

[2] J.C. Artés, J. Llibre, J.C. Medrado and M.A. Teixeira, Piecewise linear differential systems with two real saddles, Math. Comput. Simul. 95 (2013), 13-22.

[3] R. Benterki AND J. Llibre, The limit cycles of discontinuous piecewise linear differential systems formed by centers and separated by irreducible cubic curves I, to appear in Dynamics of Continuous, Discrete and Impulsive Systems-Series A, 2020.

[4] R. Benterki AND J. Llibre, The limit cycles of discontinuous piecewise linear differential systems formed by centers and separated by irreducible cubic curves II, accepted to be published, 2020.

[5] R.Benterki ANd J.LLibre, Crossing Limit Cycles of Planar Piecewise Linear Hamiltonian Systems without Equilibrium Points. Mathematics 2020, 8, 755.

[6] M. di Bernardo, C.J. Budd, A.R. Champneys And P. KowalczyK, Piecewise-Smooth Dynamical Systems: Theory and Applications, Appl. Math. Sci., vol. 163, Springer-Verlag, London, 2008.

[7] R. Bix, Conics and cubics, Undergraduat Texts in Mathematics, Second Edition, Springer, 2006.

[8] D.C. BRAGA AND L.F.Mello, Limit cycles in a family of discontinuous piecewise linear differential systems with two zones in the plane, Nonlinear Dynam. 73 (2013) 128-1288.

[9] R.D. Euzébio AND J. Llibre, On the number of limit cycles in discontinuous piecewise linear differential systems with two pieces separated by a straight line, J. Math. Anal. Appl. 424(1) (2015), 475-486.

[10] A.F. Fonseca, J. Llibre ANd L.F. Mello, Limit cycles in planar piecewise linear Hamiltonian systems with three zones without equilibrium points, to appear in Int. J. Bifurcation and Chaos, 2020.

[11] E. Freire, E. Ponce, F. Rodrigo AND F. Torres, Bifurcation sets of continuous piecewise linear systems with two zones, Int. J. Bifurcation and Chaos 8 (1998), 2073-2097.

[12] E. Freire, E. Ponce And F. Torres, Canonical discontinuous planar piecewise linear systems, SIAM J. Appl. Dyn. Syst. 11(1)(2012), 181-211.

[13] J.J. Jimenez, J. Llibre And J.C. Medrado, Crossing limit cycles for a class of piecewise linear differential centers separated by a conic, Preprint, 2019.

[14] J. Llibre, D.D. Novaes AND M.A. TeIxeIRA, Limit cycles bifurcating from the periodic orbits of a discontinuous piecewise linear differential center with two zones, Int. J. Bifurcation and Chaos 25 (2015), 1550144, pp. 11. 
[15] J. Llibre And E. Ponce, Piecewise linear feedback systems with arbitrary number of limit cycles, Internat. J. Bifur. Chaos Appl. Sci. Engrg. 13 (2003), 895-904.

[16] J. Llibre, E. Ponce AND X. Zhang, Existence of piecewise linear differential systems with exactly $n$ limit cycles for all $n \in \mathbb{N}$, Nonlinear Anal.54 (2003) 977-994.

[17] J. Llibre And E. Ponce, Three nested limit cycles in discontinuous piecewise linear differential systems with two zones, Dyn. Contin. Discr. Impul. Syst., Ser. B 19 (2012), 325-335.

[18] J. Llibre And M.A. Teixeira, Piecewise linear differential systems with only centers can create limit cycles ?, Nonlinear Dyn. 91 (2018), 249-255.

[19] J. Llibre AND X. Zhang, Limit cycles for discontinuous planar piecewise linear differential systems separated by an algebraic curve, Int. J. Bifurcation and Chaos 29 (2019), 1950017, pp. 17.

[20] O. MaKarenKov And J.S.W. Lamb, Dynamics and bifurcations of nonsmooth systems: a survey, Phys. D 241 (2012), 1826-1844.

[21] D.J.W. Simpson, Bifurcations in Piecewise-Smooth Continuous Systems, World Sci. Ser. Nonlinear Sci. Ser. A, vol. 69, World Scientific, Singapore, 2010. 